\title{
Spectral Analysis of Quadrature Rules and Fourier Truncation-based Methods Applied to Shading Integrals
}

\author{
Ricardo Marques, Christian Bouville and Kadi Bouatouch
}

\begin{abstract}
We propose a theoretical framework, based on the theory of Sobolev spaces, that allows for a comprehensive analysis of quadrature rules for integration over the sphere. We apply this framework to the case of shading integrals in order to predict and analyze the performances of quadrature methods. We show that the spectral distribution of the quadrature error depends not only on the samples set size, distribution and weights, but also on the BRDF and the integrand smoothness. The proposed spectral analysis of quadrature error allows for a better understanding of how the above different factors interact. We also extend our analysis to the case of Fourier truncation-based techniques applied to the shading integral, so as to find the smallest spherical/hemispherical harmonics degree $L$ (truncation) that entails a targeted integration error. This application is very beneficial to global illumination methods such as Precomputed Radiance Transfer and Radiance Caching. Finally, our proposed framework is the first to allow a direct theoretical comparison between quadrature- and truncation-based methods applied to the shading integral. This enables, for example, to determine the spherical harmonics degree $L$ which corresponds to a quadrature-based integration with $\mathrm{N}$ samples. Our theoretical findings are validated by a set of rendering experiments.
\end{abstract}

Index Terms-Rendering Equation, Spectral Analysis, Monte Carlo Methods, Spherical Harmonics Decomposition.

\section{INTRODUCTION}

$\mathrm{R}$ ECENT research works have shown the interest in the Fourier analysis of rendering algorithms (e.g. Belcour et al. [4] and Mehta et al. [22]), and especially with regard to sampling strategies (e.g. Ramamoorthi et al. [25], Subr and Kautz [29], and Pilleboue et al. [24]). However, most research works are focused on the general case of Monte Carlo methods and do not consider a spectral analysis of spherical quasi-Monte Carlo (QMC) techniques or, more specifically, deterministic hemispherical quadrature rules applied to illumination integrals. This is, in our opinion, an important gap in the literature as QMC methods are currently widespread in computer graphics as shown by Keller [15]. Another integration technique, commonly used in rendering applications, consists in computing the integral in the spectral domain. In this case, the integration error results from the truncation of the theoretically infinite spectrum of the integrand. Our goal in this paper is thus to propose a theoretical framework for (i) a spectral analysis of hemispherical quadrature techniques for evaluating the shading integral, and (ii) an analysis of the error due to the truncation of the spherical harmonics expansion of the integrand. In both cases, we use the theory of Sobolev spaces to model the smoothness characteristics of the integrand as explained below.

It is well known that the error bounds of QMC techniques strongly depend on the smoothness of the integrand. Compared to a classic Monte Carlo method, no useful error bounds can be found if the integrand is highly discontinuous. For analyzing the performances of quadrature rules, we have based our approach on

- R. Marques is with Universitat Pompeu Fabra, Grup de Recerca de Tecnologies Interactives, Barcelona, Spain.

E-mail: ricardo.marques@upf.edu

- C. Bouville and K. Bouatouch are with IRISA, University of Rennes 1, Rennes, France.

Manuscript received MONTH DAY, 2018; revised MONTH DAY, 2019.
Sobolev spaces theory. Sobolev spaces are already broadly used in the theory of quadrature rules on the sphere (e.g., Brauchart et al. [7]). They provide an elegant mathematical framework to characterize functions smoothness in the spectral domain.

The main points of our contribution concern: (i) the spectral analysis of spherical / hemispherical quadrature rules with the new notions of zonal and anisotropic errors; (ii) the spectral analysis of the spherical / hemispherical harmonic $(\mathrm{SH} / \mathrm{HSH}$, respectively) expansion of the shading integral, and the effect of truncating this expansion on the evaluation of shading integrals; (iii) a mathematical framework for analyzing the performance of quadrature rules and of the truncation of the $\mathrm{SH} / \mathrm{HSH}$ expansion of shading integrals in terms of worst-case error and spectral distribution of the integration error. We apply the theoretical framework proposed in this paper to various QMC techniques so as to analyze and predict their performances. We also apply it to the case of Fourier truncation-based techniques for computing the shading integral. This spectral framework allows finding the smallest $\mathrm{SH} / \mathrm{HSH}$ degree $\mathrm{L}$ that entails a targeted integration error, which is very beneficial to global illumination methods such as precomputed radiance transfer (PRT) [27] and radiance caching (RC) [17], or to quadrature-based techniques such as quasi-Monte Carlo (QMC).

In the following, after a presentation of related works in this domain (Sec. 2), we will briefly introduce the necessary mathematical background on the spectral analysis of spherical functions and the theory of Sobolev spaces (Sec. 3). Then, our contribution begins with the spectral analysis of the main types of illumination integrals (Sec. 4) followed by a spectral analysis of integration errors produced by quadrature rules (Sec. 5) and by the truncation of the shading integral in the Fourier domain. Our theoretical framework is then applied to the theoretical analysis of currently-used QMC methods (Sec. 7), and of the truncation in the Fourier domain (Sec. 8). In Sec. 6 we provide formal proofs of the 
most important equations given in the paper. In Sec. 9 we show that our theoretical analysis is in accordance with experimental data. Then we conclude in Sec. 10.

\section{Related WORK}

The spectral analysis of the shading integral was first studied in the context of the inverse rendering problem [3], [23], [26]. Although these papers propose a thorough spherical harmonics analysis of common BRDFs, none of these papers addresses the issues related to integral approximation and incident radiance sampling. With regard to spectral analysis of stochastic sampling, Subr and Kautz [29] give an extensive review of the literature on this subject and propose new quality measures of sampling strategies. Pilleboue et al. [24] propose a Fourier analysis of stochastic Monte-Carlo integration on the sphere. Their approach is based on a stochastic analysis of the sampling process whereas our approach is essentially deterministic. The difference between both methods is analyzed in details at the end of this section. Most of the literature on quadrature methods on the sphere, and on spherical QMC methods in particular, resorts to a reproducing kernel Hilbert spaces (RKHS) approach originally proposed by Hickernell [14]. Under some restrictions, Sobolev spaces are RKHSs. Sobolev spaces are function spaces that have particularly interesting properties to model functions of varying smoothness. This theory has been extensively used to analyze quadratures on the sphere (e.g. Brauchart and Dick [5]) and it can be used in many different ways to optimize point sets distribution on the sphere (see, e.g., Brauchart and Grabner [6] and Brauchart et al. [7]). Marques et al. [19] use the theory of Sobolev spaces to derive optimal sample weights (OSW) for any samples distribution on the hemisphere. They apply their method to the computation of shading integrals and show that their method outperforms all familiar methods used in this context.

In this paper, our approach to integration quadrature error is essentially deterministic. As opposed to the method of Pilleboue et al. [24] which is based on a stochastic Monte Carlo analysis, in our approach the point distribution is not necessarily assumed to be random which allows a spectral analysis of quadrature error due to a single (fixed) point set. Moreover, in our approach, the analyzed sample set need not be homogeneous (according to the definition given by Pilleboue et al. [24]) as our framework can deal with any sample distribution. Besides our quadrature analysis is not limited to simple samples averaging estimate, as in stochastic Monte Carlo or in basic QMC, but also deals with the most general form of weighted average estimation. Furthermore, our model considers integrands as the product of two functions. The first one represents the incident radiance function which is supposed to be unknown and the second one represents the BRDF (either in the exact or in an approximate form). In this way, our approach can easily factor in the effect of importance sampling which basically consists in morphing the sample sets so as to adapt to the shape of the BRDF. This integrand model thus allows a spectral analysis of quadrature error in application cases that correspond quite closely to computer graphics practices. Our theoretical framework directly deals with a hemispheric integration domain without any use of coordinate transformations and no particular symmetry property is required for the integrand as in Pilleboue et al. [24]. Finally, our error analysis is not based on particular profiles of integrand power spectra but on a more general Sobolev space model of the integrand, which allows a spectral analysis in a wide range of integrand smoothness. Furthermore, we show that the Sobolev spaces approach is not limited to the analysis of point sampling quadrature but can also be extended to frequency domain integration as explained below. This enables us to compare both integration methods on the same basis. Indeed, another commonlyused approach to estimate the shading integral is to perform the inner product between the incident radiance function and the BRDF function in the spherical harmonics domain. The integral approximation is then obtained by computing a truncated $\mathrm{SH}$ expansion of this inner product up to a degree $L$.

Our objective is to propose a spectral analysis of the error resulting from the approximation of shading integrals with a classic quadrature or with an inner product truncation method. With this analysis it is possible to determine the maximum degree $\mathrm{L}$ of the $\mathrm{SH}$ expansion of the incidence radiance function so as to get an acceptable integration error with an inner product truncation method. For both classic quadrature and inner product truncation, we use the same theoretical approach based on Sobolev spaces.

\section{Mathematical background ON SPHERICAL FUNCTIONS}

\subsection{Spectral analysis of spherical functions}

In this subsection and the following one, we briefly introduce the mathematical background about spectral analysis of spherical functions by means of Legendre spherical harmonics $(\mathrm{SH})$ and hemispherical harmonics (HSH). For more information on this subject, we refer the reader to Freeden and Gervens [10] and to Atkinson and Han [2]. SH basis functions are defined on the $\mathbb{S}^{2}$ sphere in $\mathbb{R}^{3}$ as follows. Given any integer $l \geq 0$ and any integer $m,|m| \leq l$, the SH $Y_{l, m}(\theta, \phi)$ of degree $l$ and order $m$ is given by:

$$
\begin{cases}Y_{l, m}(\theta, \phi)=N_{l, m} P_{l}^{m}(\cos \theta) e^{i m \phi} & \text { for } m \geq 0, \\ Y_{l, m}(\theta, \phi)=(-1)^{m} Y_{l,-m}^{*} & \text { for } m<0,\end{cases}
$$

where $(\theta, \phi)$ are the spherical coordinates of a point on $\mathbb{S}^{2}$ and $x^{*}$ denotes the complex conjugate of $x . N_{l, m}$ is a normalization coefficient that makes the $\mathrm{SH}$ orthonormal:

$$
N_{l, m}=\sqrt{\frac{2 l+1}{4 \pi} \frac{(l+m) !}{(l-m) !}}
$$

and $P_{l}^{m}(\cos \theta)$ are the associated Legendre functions which become Legendre polyomials $P_{l}(\cos \theta)$ for $m=0$. In the following, we will also make use of the $\mathrm{SH}$ addition theorem:

$$
\sum_{m=-l}^{m=l} Y_{l, m}(\mathbf{u}) Y_{l, m}^{*}(\mathbf{v})=\frac{2 l+1}{4 \pi} P_{l}(\mathbf{u} \cdot \mathbf{v}), \quad \mathbf{u}, \mathbf{v} \in \mathbb{S}^{2},
$$

where $\mathbf{u} \cdot \mathbf{v}=\cos \theta$. As the shading integral is an integral over a hemisphere, the rendering applications often use hemispherical harmonics (HSH) instead of spherical harmonics [11]. HSH are derived from $\mathrm{SH}$ using the shifting property of Legendre polynomials, which leads to the following definition of the shifted associated Legendre functions $\tilde{P}_{l}^{m}$ :

$$
\tilde{P}_{l}^{m}(x)=P_{l}^{m}(2 x-1) \quad \text { with } \quad x \in[0,1] .
$$

Based on this definition, the hemispherical harmonics basis functions (HSH) can be defined as follows:

$$
\tilde{Y}_{l, m}(\theta, \phi)=\tilde{N}_{l, m} \tilde{P}_{l}^{m}(\cos \theta) e^{i m \phi},
$$

with $\theta \in[0, \pi / 2], \phi \in[0,2 \pi]$ and the normalization coefficient:

$$
\tilde{N}_{l, m}=\sqrt{\frac{2 l+1}{2 \pi} \frac{(l+m) !}{(l-m) !}} .
$$


HSH coefficients can be converted to $\mathrm{SH}$ coefficients and vice versa as explained by Gautron et al. [11]. However, when converting from $\mathrm{HSH}$ to $\mathrm{SH}$, the reconstructed spherical function will be zero-valued in the lower hemisphere. Note that SH have to be used when considering participatiing media and environments maps.

\subsection{Sobolev spaces on the $\mathbb{S}^{2}$ sphere}

As quadrature error depends on the integrand smoothness, it is necessary to characterize this factor in a way that can be easily handled in both original and spectral domains. Sobolev spaces for spherical functions are well-suited to this requirement [2], [10]. They are function spaces, denoted by $\mathbb{H}^{s}\left(\mathbb{S}^{2}\right)$, whose smoothness is characterized by a parameter $s \in \mathbb{R}^{+}$. The larger the value of $s$, the smoother the spherical functions belonging to $\mathbb{H}^{s}\left(\mathbb{S}^{2}\right)$. Furthermore, as we show below, Sobolev spaces also have the advantage of allowing a characterization of the function space in the spectral domain, which will be very useful for the spectral analysis of integration error addressed in this paper.

When the Sobolev space coefficient $s=0$, then we have $\mathbb{H}^{0}\left(\mathbb{S}^{2}\right)=\mathbb{L}_{2}\left(\mathbb{S}^{2}\right)$, i.e., the space of square integrable functions. When $s>1$, all functions $f \in \mathbb{H}^{s}\left(\mathbb{S}^{2}\right)$ are bounded. As explained by Marques et al. [19], it is an essential property to have a bounded quadrature evaluation, which is strictly required for the error analysis performed in the remainder of this paper. Therefore, only the Sobolev spaces with $s>1$ are considered in the following. In this case, Sobolev spaces are reproducing kernel Hilbert spaces (RKHS) [13], which means that given a Sobolev space $\mathbb{H}^{s}\left(\mathbb{S}^{2}\right)$, there exists a kernel $K^{(s)}(\mathbf{u}, \mathbf{v})$ such that $K^{(s)}(\cdot, \mathbf{v}) \in \mathbb{H}^{s}\left(\mathbb{S}^{2}\right)$ and:

$$
\left\langle f(\cdot), K^{(s)}(\cdot, \mathbf{v})\right\rangle_{\mathbb{H}^{s}}=f(\mathbf{v}), \quad \mathbf{v} \in \mathbb{S}^{2}, f \in \mathbb{H}^{s}\left(\mathbb{S}^{2}\right),
$$

the latter being known as the reproducing property. Note that this equation holds because $f(\mathbf{v})$ is always bounded. In the following, we assume that the kernel $K^{(s)}(\mathbf{u}, \mathbf{v})$ is isotropic, i.e. $K^{(s)}(\mathbf{u}, \mathbf{v})=K^{(s)}(\mathbf{v}, \mathbf{u})$ and $K^{(s)}(\mathbf{u}, \mathbf{v})$ only depends on the difference $\mathbf{u}-\mathbf{v}$. Thus $K^{(s)}(\mathbf{u}, \mathbf{v})$ can be expanded in terms of Legendre polynomials yielding:

$$
K^{(s)}(\mathbf{u}, \mathbf{v})=\sum_{l=0}^{\infty} a_{l}^{(s)} \frac{2 l+1}{4 \pi} P_{l}(\mathbf{u} \cdot \mathbf{v}) .
$$

Eq. (8) leads to the following expression of the $a_{l}^{(s)}$ coefficients:

$$
a_{l}^{(s)}=\int_{S^{2}} K^{(s)}(\mathbf{u}, \mathbf{v}) P_{l}(\mathbf{u} \cdot \mathbf{v}) d S(\mathbf{u}),
$$

where the integral has the same value for any point $\mathbf{v} \in \mathbb{S}^{2}$ because $K^{(s)}(\mathbf{u}, \mathbf{v})$ is isotropic. The kernel in Eq. (8) is thus real-valued, symmetric $\left(K^{(s)}(\mathbf{u}, \mathbf{v})=K^{(s)}(\mathbf{v}, \mathbf{u})\right)$ and isotropic. It is also a radial function as the distance $|\mathbf{u}-\mathbf{v}|$ and the inner product $(\mathbf{u} \cdot \mathbf{v})$ are related by the equation:

$$
|\mathbf{u}-\mathbf{v}|^{2}=2-2(\mathbf{u} \cdot \mathbf{v}), \text { with } \mathbf{u}, \mathbf{v} \in \mathbb{S}^{2} .
$$

Eq. (8) can be rewritten in terms of $\mathrm{SH}$ basis functions as follows [16], by application of the SH addition theorem (Eq. (3)):

$$
K^{(s)}(\mathbf{u}, \mathbf{v})=\sum_{l=0}^{\infty} a_{l}^{(s)} \sum_{m=-l}^{m=l} Y_{l, m}(\mathbf{u}) Y_{l, m}^{*}(\mathbf{v}), \quad \mathbf{u}, \mathbf{v} \in \mathbb{S}^{2},
$$

hence allowing to have an explicit SH expansion of the frequency of the Sobolev space kernel $K^{(s)}(\mathbf{u}, \mathbf{v})$. Note that the spectral features of the function space are controlled by the $a_{l}^{(s)}$ coefficients which, as noted by Brauchart et al. [7] exhibit a rate of decay in
$O\left(l^{-2 s}\right)$, with $s$ being the smoothness parameter. As Sobolev spaces are Hilbert spaces then, given any functions $f, g \in \mathbb{H}^{s}\left(\mathbb{S}^{2}\right)$, there exists an inner product $\langle f, g\rangle_{\mathbb{H}^{s}}$ defined by:

$$
\langle f, g\rangle_{\mathbb{H}^{s}}:=\sum_{l=0}^{\infty} \sum_{m=-l}^{m=l} \frac{1}{a_{l}^{(s)}} f_{l, m} g_{l, m}^{*},
$$

where $f_{l, m}$ and $g_{l, m}$ are the SH coefficients of $f$ and $g$ respectively. The inner product in Eq. (12) induces the norm $\|f\|_{\mathbb{H}^{s}}$ such that:

$$
\|f\|_{\mathbb{H}^{s}}:=\left(\sum_{l=0}^{\infty} \sum_{m=-l}^{m=l} \frac{1}{a_{l}^{(s)}}\left|f_{l, m}\right|^{2}\right)^{\frac{1}{2}} .
$$

As explained by Brauchart et al. [7], there exists some degree of freedom in choosing the kernel $K^{(s)}(\mathbf{u}, \mathbf{v})$ since all positive definite isotropic kernels (i.e. $a_{l}^{(s)}>0$ ) have equivalent Sobolev space norms as long as the rate of decay of their $a_{l}^{(s)}$ coefficients is in $O\left(l^{-2 s}\right)$. In the following, we use the generalized distance kernel [7] because it has the advantage of having an analytic form. When $1<s<2$, this kernel is defined as follows:

$$
K_{g d}^{(s)}(\mathbf{u}, \mathbf{v})=\frac{2^{2 s-1}}{s}-|\mathbf{u}-\mathbf{v}|^{2 s-2},
$$

and the $a_{l}^{(s)}$ coefficients are given by:

$$
\begin{aligned}
& a_{0}^{(s)}=4 \pi \frac{2^{2 s-2}}{s}, \\
& a_{l}^{(s)}=-4 \pi \frac{2^{2 s-2}}{s} \frac{\Gamma(1-s+l) \Gamma(1+s)}{\Gamma(1+s+l) \Gamma(1-s)}, \quad l \geq 1 .
\end{aligned}
$$

where $\Gamma$ (.) represents the extended factorial Gamma function. Note that the kernel $K^{(s)}(\mathbf{u}, \mathbf{v})$ as defined by Eq. (11) has an infinite spectral bandwidth and consequently kernel-based approximation with this kernel also generates functions with infinite spectral bandwidth. This property enables to approximate any function in $\mathbb{L}_{2}\left(\mathbb{S}^{2}\right)$ with arbitrary precision (i.e. Sobolev spaces are dense in $\mathbb{L}_{2}\left(\mathbb{S}^{2}\right)$ ). This property is not met with classic band-limited approximation methods, hence making it more appropriate to represent the potentially sharp variations of the incident radiance functions. Furthermore, as shown later by our experimental results (Sec. 9.1), we have observed that many incident radiance functions are optimally modeled by functions with a smoothness close to $s=1.5$.

\section{A SPECTRAL ANALYSIS FRAMEWORK FOR SHADING INTEGRALS}

\subsection{General case}

In shading integrals, the integrand is basically the product of two spherical functions: the incident radiance $f(\mathbf{u})$ and a weight function $p(\mathbf{u})$ which corresponds to the product $\mathrm{BRDF} \times \cos (\theta)$, $\theta$ being the incidence angle. In the following, we consider that $p(\mathbf{u}) \in \mathbb{L}^{2}$ is an analytically known function which is non-zero only over the upper hemisphere $H_{z+}$ aligned with the $\mathbf{z}$ axis, which means that $p(\mathbf{u})=0$ if $\mathbf{u} \in H_{z-}$ (i.e., when $\mathbf{u} \cdot \mathbf{z}=\cos \theta<0$ ). The incident radiance function $f(\mathbf{u})$ is assumed to belong to some Sobolev space $\mathbb{H}^{s}\left(\mathbb{S}^{2}\right)$. So as to facilitate the connection with QMC theory, we normalize the shading integral as follows:

$$
I_{f}=\frac{1}{2 \pi r_{s}} \int_{S^{2}} f(\mathbf{u}) p(\mathbf{u}) d S(\mathbf{u}),
$$

where the normalization coefficient $r_{s}$ is:

$$
r_{s}=\frac{1}{2 \pi} \int_{S^{2}} p(\mathbf{u}) d S(\mathbf{u})
$$


taking into account that $p(\mathbf{u})=0$ in the lower hemisphere $H_{z-}$. In the cases studied hereafter, the BRDF is a zonal function (i.e. its value depends only on the polar angle of the incident direction), that is, $p(\mathbf{u}):=p(\mathbf{u} \cdot \mathbf{z})=p(\cos \theta)$, in which case its SH coefficients $p_{l, m}=0$ if $m \neq 0$. If the BRDF is not analytically known or is anisotropic, it can be substituted with an analytical approximation by replacing $f(\mathbf{u})$ with $f^{\prime}(\mathbf{u})=f(\mathbf{u}) p(\mathbf{u}) / p^{\prime}(\mathbf{u})$ where $p^{\prime}(\mathbf{u})$ is a zonal approximation of $p(\mathbf{u})$. Thus, in application of the inner product property (Eq. (12)), we have:

$$
I_{f}=\frac{1}{2 \pi r_{s}} \int_{S^{2}} f(\mathbf{u}) p(\mathbf{u}) d S(\mathbf{u})=\sum_{l=0}^{\infty} f_{l, 0} b_{l}^{(p)},
$$

with:

$$
b_{l}^{(p)}=\frac{\left\langle p, Y_{l, 0}\right\rangle_{\mathbb{L}^{2}}}{2 \pi r_{s}}=\frac{1}{2 \pi r_{s}} \int_{S^{2}} p(\mathbf{u}) Y_{l, 0}(\mathbf{u}) d S(\mathbf{u}),
$$

where the $b_{l}^{(p)}$ coefficients are the zonal harmonics coefficients of the normalized weight function $p(\mathbf{u})$. In the above equations, recall that the hemispherical nature of the integration domain is conditioned by the weight function $p(\mathbf{u})$ whose value is set to zero in the lower hemisphere $H_{z_{-}}$. In the case HSH are used instead of SH, equivalent equations to Eq. (19) and (20) are obtained by simply replacing SH $Y_{l, 0}$ basis functions by HSH $\tilde{Y}_{l, 0}$ basis functions and $b_{l}^{(p)}, f_{l, 0} \mathrm{SH}$ coefficients by HSH coefficients $\tilde{b}_{l}^{(p)}, \tilde{f}_{l, 0}$. In the following, two basic cases of shading integrals are analyzed, i.e. diffuse and glossy reflections.

\subsection{Diffuse reflection case}

In the case of diffuse reflection, the weight function $p(\mathbf{u})=(\mathbf{u} \cdot \mathbf{z})$, $\forall \mathbf{u} \in H_{z+}$ and $p(\mathbf{u})=0$ elsewhere. Then $r_{s}=1 / 2$ and using Eq. (20), the SH coefficients of the normalized weight function for the diffuse reflection case $b_{l}^{(p)}=b_{l}^{(d)}$ are:

$$
b_{l}^{(d)}=\sqrt{\frac{(2 l+1)}{\pi}} \int_{0}^{\frac{\pi}{2}} P_{l}(\cos \theta) \cos \theta \sin \theta d \theta .
$$

This equation can be expressed analytically as follows (cf. Basri and Jacobs [3] or Ramamoorthi and Hanrahan [26]):

$$
b_{l}^{(d)}= \begin{cases}(-1)^{\frac{l}{2}+1} \sqrt{\frac{(2 l+1)}{\pi}} \frac{1}{2^{l}(l-1)(l+2)} \frac{l !}{\left[\left(\frac{l}{2}\right) !\right]^{2}} & \text { if } l>1 \text { even, } \\ 0 & \text { if } l>1 \text { odd, } \\ \frac{1}{\sqrt{3 \pi}} & \text { if } l=1, \\ \frac{1}{2 \sqrt{\pi}} & \text { if } l=0 .\end{cases}
$$

As for the HSH case, Eq. (21) becomes:

$$
\tilde{b}_{l}^{(d)}=\sqrt{\frac{2(2 l+1)}{\pi}} \int_{0}^{\frac{\pi}{2}} \tilde{P}_{l}(\cos \theta) \cos \theta \sin \theta d \theta .
$$

As $\tilde{P}_{l}(z)=P_{l}(2 z-1)$, we can use the variable substitution $z^{\prime}=$ $2 z-1=2 \cos \theta-1$ to get:

$$
\tilde{b}_{l}^{(d)}=\sqrt{\frac{2(2 l+1)}{\pi}} \frac{1}{4} \int_{-1}^{1}\left(z^{\prime}+1\right) P_{l}\left(z^{\prime}\right) d z^{\prime} .
$$

The integral in Eq. (24) can be interpreted as the projection of the first degree $z^{\prime}+1$ polynomial on the basis of Legendre polynomials that are orthogonal on the $[-1,1]$ interval with respect to the $\mathbb{L}^{2}$ norm. Then, only the first two coefficients $\tilde{b}_{0}^{(d)}=1 / \sqrt{2 \pi}$ and $\tilde{b}_{1}^{(d)}=$ $1 / \sqrt{6 \pi}$ are non-zero.

\subsection{Glossy reflection case}

For the glossy reflection case, we use the Gaussian weight function:

$$
p(\mathbf{u})=e^{n\left[\left(\mathbf{u} \cdot \mathbf{u}_{r}\right)-1\right]},
$$

where $\mathbf{u}_{r}$ is the perfect mirror reflection direction and $n$ is the glossiness parameter. Then $\theta_{r}=\left(\mathbf{u}, \mathbf{u}_{r}\right)$ is the angle between the ray incidence direction $\mathbf{u} \in H_{z+}$ and $\mathbf{u}_{r}$ for a given observation direction. Gaussian weight functions of this type have been analyzed in [20] and it is shown that the normalization coefficient is $r_{s}=\left(1-e^{-n}\right) / n$. This weight function closely approximates the well-known Phong's weight function $\cos ^{n} \theta_{r}$ but the analytical expression of the $b_{l}^{(p)}$ coefficients is much simpler (details are given in the next subsections). The exact values of $b_{l}^{(p)}$ coefficients for the Phong's weight function can be found in Ramamoorthi and Hanrahan [26]. For the sake of simplicity, we assume that $n$ is large enough so that $p(\mathbf{u}) \approx 0$ outside the integration domain i.e. the upper hemisphere. This implies that our analysis is not applicable to grazing angle configurations, which can be considered marginal for high values of $n$. Given this assumption, we can take the hemisphere centered about $\mathbf{u}_{r}$ as the integration domain instead of the hemisphere centered about the surface normal. The $z$ axis orientation is then aligned with $\mathbf{u}_{r}$ (hence $\mathbf{u} \cdot \mathbf{u}_{r}=z$ ) and hence $\theta_{r}=\theta$, which makes the function $p(\mathbf{u})=p(\mathbf{u} \cdot \mathbf{z})$ zonal with axis $z$. Then, from Eq. (20) and Eq. (25), and using the variable substitution $z=\cos \theta$, the normalized weight function SH coefficients called $b_{l}^{(g)}$ (since in the glossy case $b_{l}^{(p)}=b_{l}^{(g)}$ ) are given by:

$$
b_{l}^{(g)}=\frac{1}{r_{s}} \sqrt{\frac{(2 l+1)}{4 \pi}} \int_{0}^{1} e^{n(z-1)} P_{l}(z) d z .
$$

If $n$ is large enough, $p(\mathbf{u}) \approx 0$ for $\theta>\pi / 2$. Therefore, extending the boundaries of the integral in Eq. (26) to $[-1,1]$ does not change the integral value but allows considering this integral as the coefficient of a Legendre polynomial expansion of the Gaussian function. As mentioned above, the Legendre polynomial forms an orthogonal functions basis, and the integral in Eq. (26) can then be interpreted as the projection of the Gaussian function onto a Legendre polynomial basis function. This allows using the Legendre polynomial expansion of the spherical Gaussian function such as given by Eq. 10.2.37 in [1]. Based on this equation, we have derived the following analytical approximation of the normalized $\mathrm{SH}$ coefficients of the weight function:

$$
b_{l}^{(g)} \approx \frac{e^{-n}}{1-e^{-n}} \sqrt{n v} I_{v}(n), \quad \text { with } \quad v=l+\frac{1}{2},
$$

$I_{v}(x)$ being the modified Bessel function of the first kind. In the HSH expansion case, we have:

$$
\tilde{b}_{l}^{(g)}=\frac{1}{r_{s}} \sqrt{\frac{(2 l+1)}{2 \pi}} \int_{0}^{1} e^{n(z-1)} P_{l}(2 z-1) d z,
$$

which after the $z^{\prime}=2 z-1$ variable substitution gives:

$$
\tilde{b}_{l}^{(g)}=\frac{1}{2 r_{s}} \sqrt{\frac{(2 l+1)}{2 \pi}} \int_{-1}^{1} e^{\frac{n}{2}\left(z^{\prime}-1\right)} P_{l}\left(z^{\prime}\right) d z^{\prime} .
$$

We can then proceed the same way as for Eq. (26) to get:

$$
\tilde{b}_{l}^{(g)} \approx \frac{e^{\frac{-n}{2}}}{1-e^{-n}} \sqrt{n v} I_{v}\left(\frac{n}{2}\right), \text { with } v=l+\frac{1}{2}
$$

Note however, that unlike Eq. (27), we do not need to extend the integration domain to develop the integral in Eq. (30) and this expression is thus exact at normal incidence. 


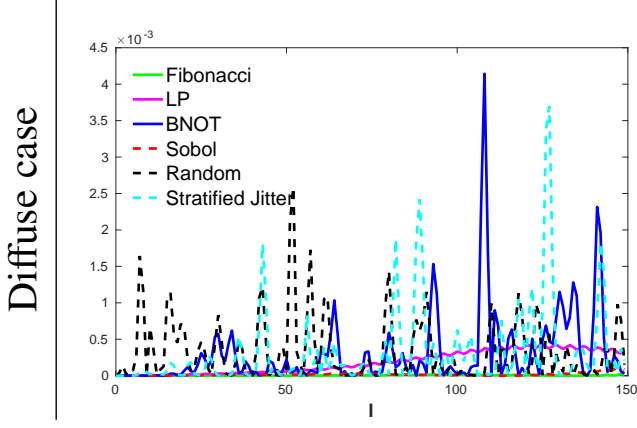

(a) $d_{l}^{2}$ PSCs (equal weights)

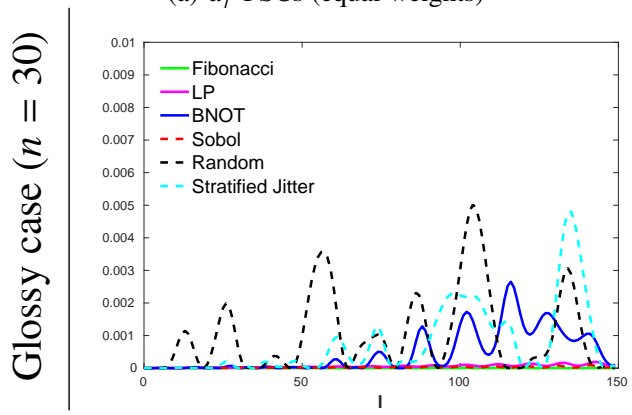

(d) $d_{l}^{2}$ PSCs (equal weights)

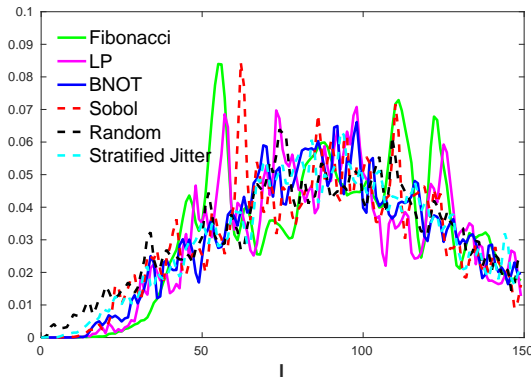

(b) $C_{I}^{2}$ PSCs (equal weights)

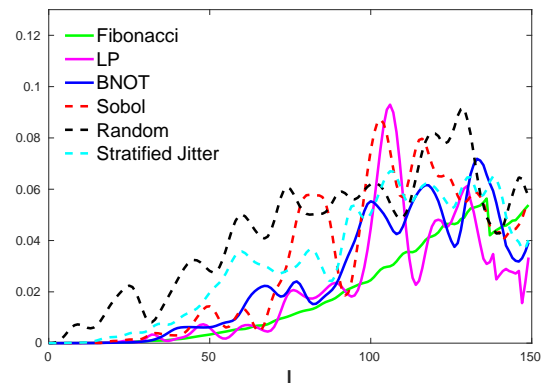

(e) $C_{l}^{2}$ PSCs (equal weights)

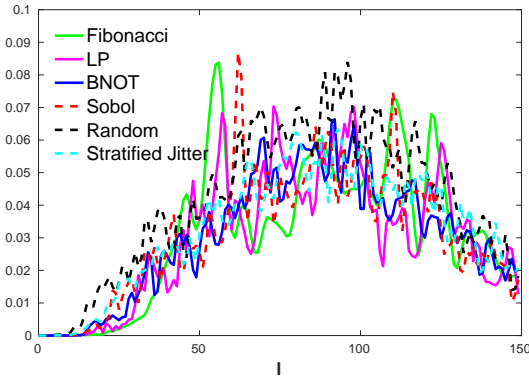

(c) $C_{1}^{2}$ PSCs (optimal weights, OSW)

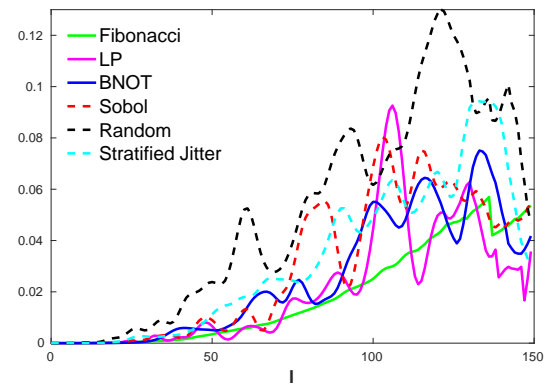

(f) $C_{l}^{2}$ PSCs (optimal weights, OSW)

Fig. 1. Power spectrum of quadrature rules using different point sets as a function of the expansion degree $l$. A single point set realization containing 256 samples was used for each considered point set generator. The top and bottom rows depict the diffuse and glossy reflection cases, respectively. The shininess coefficient for the glossy reflection case is $n=30$. The left column shows the zonal power spectrum coefficients (PSC) $d_{l}^{2}$ for equal-weights quadrature rules. The center column shows the anisotropic PSCs $C_{l}^{2}$ for equal-weights quadrature rules. The right column shows the anisotropic PSCs $C_{l}^{2}$ for optimal-weights quadrature rules (Marques et al. [19]). Note that the zonal coefficients $d_{l}^{2}$ are roughly two orders of magnitude smaller than the anisotropic $C_{l}^{2}$ coefficients, making the worst-case error $E^{(s)}$ in Eq. (42) be dominated by the anisotropic error $E_{Z}^{(s)}$ (see Eqs. (46) and (47)). Note also that the effect of the optimal sample weights (OSW) in reducing the value of the $C_{l}^{2}$ power spectrum coefficients (PSCs) at low frequencies, which is particularly visible for random point sets. Refer to Fig. 2 for a clearer comparison of the low frequency spectra in the diffuse case.

\subsection{Conversions from HSH to SH}

As the $\mathrm{SH}$ and $\mathrm{HSH}$ expansion of $p(\mathbf{u})$ and $p^{\prime}(\mathbf{u})$ only involves zonal harmonics, only conversions between zonal coefficients are relevant. Thus, given a truncated set of $L^{\prime}$ coefficients $\tilde{b}_{l^{\prime}}^{(p)}$ taken from the full HSH expansion of $p(\mathbf{u})$, the converted SH coefficients $b_{l}^{(p)}$ of $p^{\prime}(\mathbf{u})$ can be expressed by [11]:

$$
b_{l}^{(p)}=\sum_{l^{\prime}=0}^{L^{\prime}} \tilde{b}_{l^{\prime}}^{(p)} M_{l^{\prime} l}
$$

where the $M_{l^{\prime} l}$ are the conversion matrix coefficients:

$$
M_{l^{\prime} l}=\int_{S^{2}} \tilde{Y}_{l^{\prime}, 0}(\mathbf{u}) Y_{l, 0}(\mathbf{u}) d S(\mathbf{u})
$$

which can be developed as follows using Eqs. (4) and (5), and applying the $z=\cos \theta$ variable substitution:

$$
M_{l^{\prime} l}=\sqrt{\frac{(2 l+1)\left(2 l^{\prime}+1\right)}{2}} \int_{0}^{1} P_{l^{\prime}}(2 z-1) P_{l}(z) d z
$$

where $P_{l}(x)$ is the Legendre polynomial of degree $l$. Note that although the number of $\tilde{b}_{l^{\prime}}^{(p)}$ HSH coefficients is truncated to $l^{\prime}=$ $L^{\prime}$, there is still a theoretically infinite number of converted $b_{l}^{\prime(p)}$ $\mathrm{SH}$ coefficients. In our implementation, the expansion of the $b_{l}^{(p)}$ coefficients has been limited to $L=170$, which has proven to be sufficient for the applications presented in this paper.

\section{Error analysis fOr the SHAdING INTEGraL}

\subsection{Worst-case error in Sobolev spaces setting}

Our goal in this section is to study the behavior of the approximation error $I_{f}-\tilde{I}_{f}$ that results from approximating the shading integral of Eq. (17) by $\tilde{I}_{f}$ with some estimation method. In the following, we consider two approximation methods. The first one is the quadrature:

$$
\tilde{I}_{f}=\sum_{j=1}^{N} w_{j} f\left(\mathbf{u}_{\mathbf{j}}\right)
$$

where $\left\{\mathbf{u}_{j}\right\} \subset H_{z+}$ is a set of sampling points and $\left\{w_{j}\right\}$ is the set of associated weights. The set of sampling points $\left\{\mathbf{u}_{j}\right\}$ can be distributed using whatever method is appropriate and particularly the well-known importance sampling method. Typically, in information-based complexity theory [30], the information brought by the samples in Eq. (34) is classified as standard information. The second method is based on Eq. (19) and consists in truncating the $\mathrm{SH} / \mathrm{HSH}$ expansion of the inner product to give:

$$
\tilde{I}_{f}=\sum_{l=0}^{L} f_{l, 0} b_{l}^{(p)},
$$

where $L$ is the maximum degree of the $\mathrm{SH} / \mathrm{HSH}$ expansion. As opposed to Eq. (34), Eq. (35) is said to use linear information, i.e., linear functionals (in this case an inner product functional).

In section 3.2, we have seen that the theory of Sobolev spaces allows characterizing the integrand smoothness under the form of a smoothness parameter $s$. We have then assumed that $f(\mathbf{u}) \in \mathbb{H}^{s}\left(\mathbb{S}^{2}\right)$ where $\mathbb{H}^{s}\left(\mathbb{S}^{2}\right)$ is a Sobolev space of smoothness 


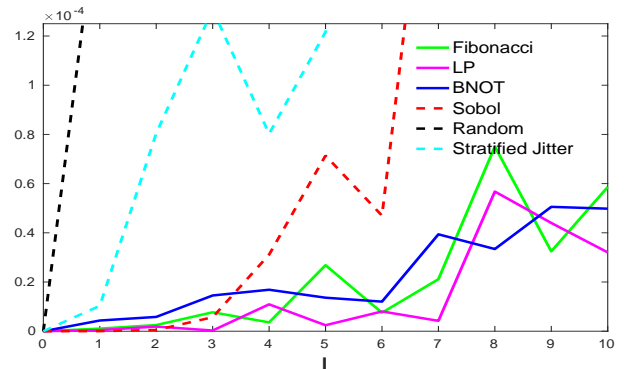

(a) $C_{l}^{2}$ PSCs (equal weights)

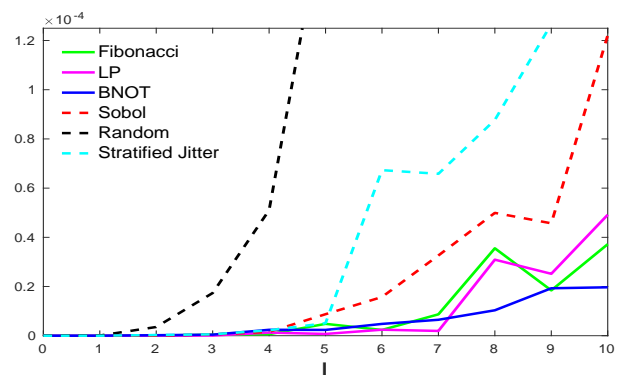

(b) $C_{l}^{2}$ PSCs (optimal weights)

Fig. 2. Close-up views of Figs. 1 (b) and (c) showing the effect of the optimal sample weights [19] on the first 10 anisotropic power spectrum coefficients (PSC) $C_{l}^{2}$. Note the significant reduction of the $C_{l}^{2}$ values in (b).

$s>1$. Under this assumption, we have seen that $\mathbb{H} \mathbb{I}^{s}\left(\mathbb{S}^{2}\right)$ is a RKHS and consequently $f(\mathbf{u})$ is bounded whatever $f \in \mathbb{H}^{s}\left(\mathbb{S}^{2}\right)$. Then, examining equations (17), (34) and (35), $I_{f}$ and $\tilde{I}_{f}$ can be considered as bounded linear functionals of $f$. The approximation error $I_{f}-\tilde{I}_{f}$ is thus also a bounded linear functional of $f$. Thus, in application of the Riesz representation theorem [2], there exists a function $h^{(s)} \in \mathbb{H}^{s}\left(\mathbb{S}^{2}\right)$ called the representer such that:

$$
I_{f}-\tilde{I}_{f}=\left\langle f, h^{(s)}\right\rangle_{\mathbb{H}^{s}},
$$

where $\tilde{I}_{f}$ is an approximation of $I_{f}$ using either a quadrature or a truncation. We can then apply the Cauchy-Schwartz inequality to obtain the following error bound:

$$
\left|I_{f}-\tilde{I}_{f}\right| \leq\|f\|_{\mathbb{H}^{s}}\left\|h^{(s)}\right\|_{\mathbb{H}^{s}}
$$

In the literature (cf. e.g. [7]), the quadrature error is usually characterized by the worst case error defined by:

$$
E^{(s)}=\sup _{f \in \mathbb{H}^{s}\left(\mathbb{S}^{2}\right),\|f\| \leq 1}\left|\tilde{I_{f}}-I_{f}\right|
$$

This definition can also be applied to the approximation method of Eq. (35). Then, using Eq. (37), we can write:

$$
E^{(s)}=\left\|h^{(s)}\right\|_{\mathbb{H}^{s}}=\left\langle h^{(s)}, h^{(s)}\right\rangle_{\mathbb{H}^{s}}^{1 / 2}
$$

Note that if $\|f\|>1$, the worst-case error is simply obtained by multiplying $E^{(s)}$ by $\|f\|$ in application of Eq. (37), which does not change the theoretical analysis presented hereafter. An interesting consequence of this formulation is that it allows to characterize the approximation error as a function of the integrand smoothness $s$. In the following, we provide an expression of the worst-case error for both aforementioned approximation methods.

\subsection{The quadrature case}

In the case of a quadrature of the form of Eq. (34), the representer of the error as defined by Eq. (36) is given by [19]:

$$
\begin{aligned}
h^{(s)}(\mathbf{u})= & \frac{1}{2 \pi r_{s}} \int_{S^{2}} K^{(s)}(\mathbf{u}, \mathbf{v}) p(\mathbf{v}) d S(\mathbf{v}) \\
& -\sum_{j=1}^{N} w_{j} K^{(s)}\left(\mathbf{u}, \mathbf{u}_{\mathbf{j}}\right) .
\end{aligned}
$$

Then, Eq. (39) can be expanded as follows:

$$
\begin{aligned}
& E^{(s)}=\left(\sum_{i=1}^{N} \sum_{j=1}^{N} w_{i} w_{j} K^{(s)}\left(\mathbf{u}_{\mathbf{i}}, \mathbf{u}_{\mathbf{j}}\right)\right. \\
& -\frac{1}{\pi r_{s}} \sum_{j=1}^{N} w_{j} \int_{S^{2}} K^{(s)}\left(\mathbf{u}_{\mathbf{j}}, \mathbf{u}\right) p(\mathbf{u}) d S(\mathbf{u}) \\
& \left.+\frac{1}{4 \pi^{2} r_{s}^{2}} \int_{S^{2}} \int_{S^{2}} K^{(s)}(\mathbf{u}, \mathbf{v}) p(\mathbf{u}) p(\mathbf{v}) d S(\mathbf{u}) d S(\mathbf{v})\right)^{1 / 2} .
\end{aligned}
$$

As noted by Marques et al. [19], some intuition can be given regarding the role of each of the above three terms in the final worst-case error $E^{(s)}$. The first term consists of a double weighted sum of evaluations of the generalized distance kernel $K^{(s)}$ for all sample pairs. Recall that the weights $w_{i}$ are the quadrature sample weights used in Eq. (34). Informally speaking, this first term penalizes irregular sample distributions, and yields a lower value to sample sets maximizing the spherical inter-samples distance. The second term is a weighted sum of integrals, where each integral accounts for the relevance of the current sample $\mathbf{u}_{j}$ with regard to the analytically-known part of the integrand $p(\mathbf{u})$. Finally, the last term is the single one which is independent of the quadrature rule and can be interpreted as highest possible worst-case error value obtained when the number of samples is $N=0$. Using the $\mathrm{SH}$ expansion of $K^{(s)}(\mathbf{u}, \mathbf{v})$ given by Eq. (11) we show in Sec. 6.1 that the worst-case error $E^{(s)}$ in Eq. (41) can be spectrally expanded as follows:

$$
E^{(s)}=\left(a_{0} d_{0}^{2}+\sum_{l=1}^{\infty} a_{l}^{(s)}\left(d_{l}^{2}+C_{l}^{2}\right)\right)^{\frac{1}{2}} .
$$

The above equation forms the core of our theoretical contribution to the spectral analysis of the error of quadrature rules. It makes the worst-case error $E^{(s)}$ emerge as a product, in the spectral domain, between two sets of coefficients: $a_{l}^{(s)}$, the spectral coefficients characterizing the assumed smoothness of the unknown part of the integrand $f(\mathbf{u})$; and $d_{l}^{2}+C_{l}^{2}$, the power spectrum coefficients characterizing the quadrature, with:

$$
d_{l}=b_{l}^{(p)}-c_{l, 0},
$$

and

$$
C_{l}^{2}=\frac{(2 l+1)}{4 \pi} \sum_{i=1}^{N} \sum_{j=1}^{N} w_{i} w_{j} P_{l}\left(\mathbf{u}_{i} \cdot \mathbf{u}_{j}\right)-c_{l, 0}^{2},
$$

with:

$$
c_{l, 0}=\sqrt{\frac{(2 l+1)}{4 \pi}} \sum_{j=1}^{N} w_{j} P_{l}\left(\mathbf{u}_{j} \cdot \mathbf{z}\right) .
$$

Recall that the $b_{l}^{(p)}$ are the SH coefficients of the normalized weight function (Eq. (20)) representing the BRDF. A closer look at Eq. (42) shows that the 'ideal' quadrature rule is the one which has the lowest possible values of the $\left(d_{l}^{2}+C_{l}^{2}\right)$ coefficients where the $a_{l}^{(s)}$ coefficients have a higher value. Since the $a_{l}^{(s)}$ coefficients 


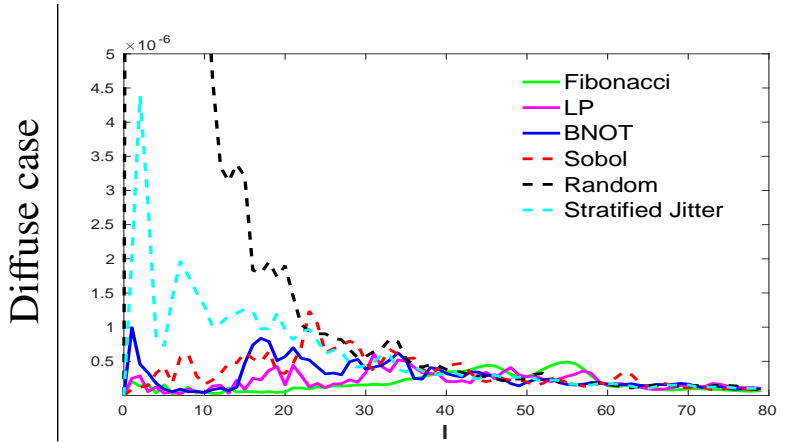

(a) Standard QMC (equal weights)

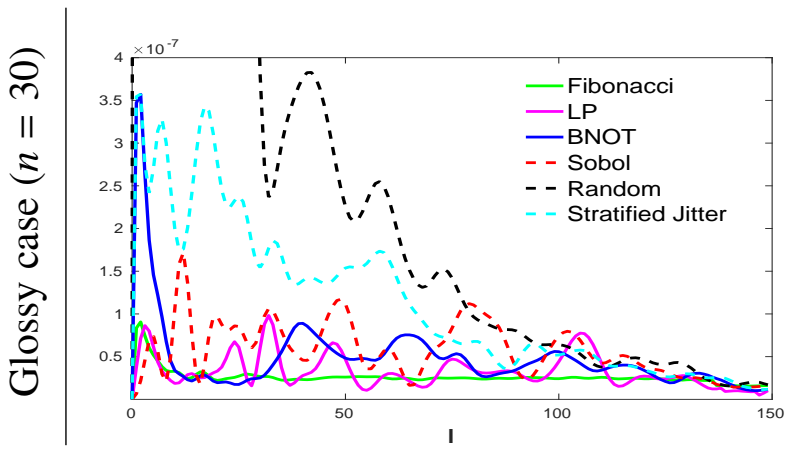

(c) Standard QMC (equal weights)

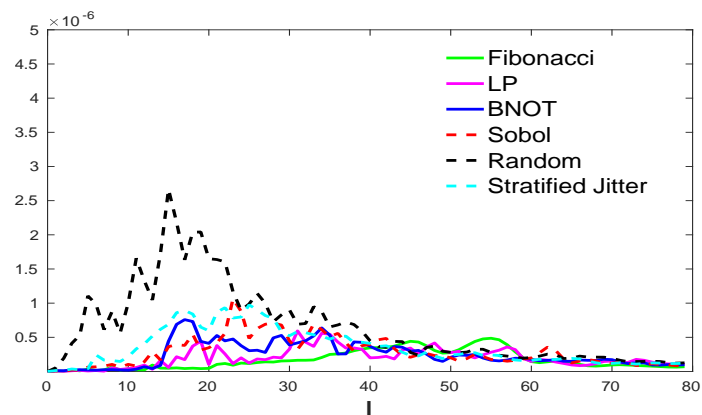

(b) With optimal sample weights (OSW)

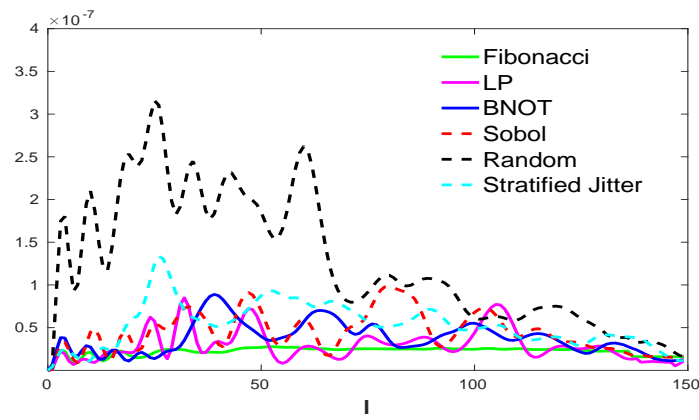

(d) With optimal sample weights (OSW)

Fig. 3. Spectral distribution of the quadrature error (Eq. (42)) as a function of the expansion degree $l$, considering that the incident radiance function $f(\mathbf{u})$ (see Eq. (17)) belongs to a Sobolev space setting with $s=1.5$. A single point set realization containing 256 samples was used for each point set generator considered. The top and bottom rows depict the diffuse and glossy reflection cases, respectively. The shininess coefficient for the glossy reflection case is $n=30$. The left column shows the spectral decomposition of the estimation error when using equal-weights QMC, while the right column shows the same when using optimal sample weights.

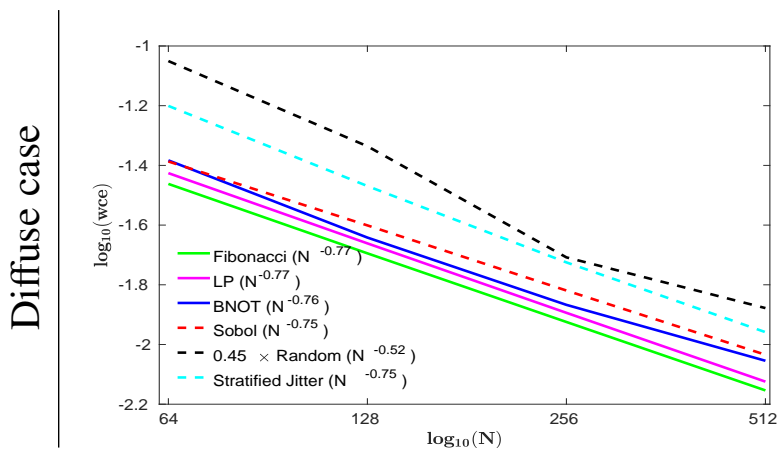

(a) Standard QMC (equal weights)

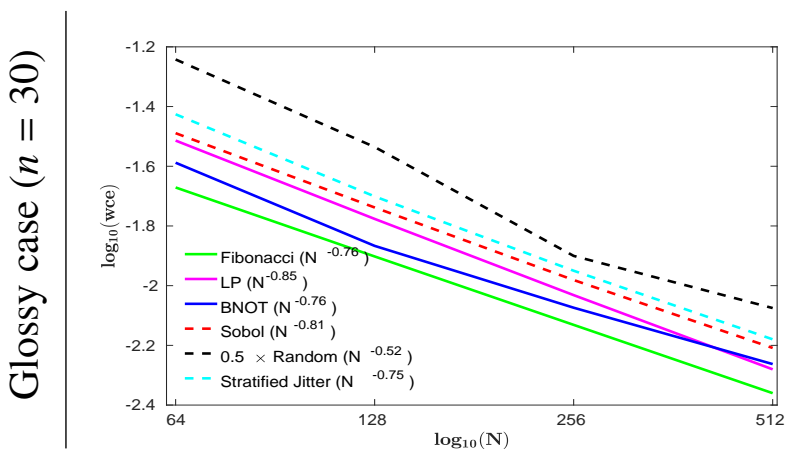

(c) Standard QMC (equal weights)

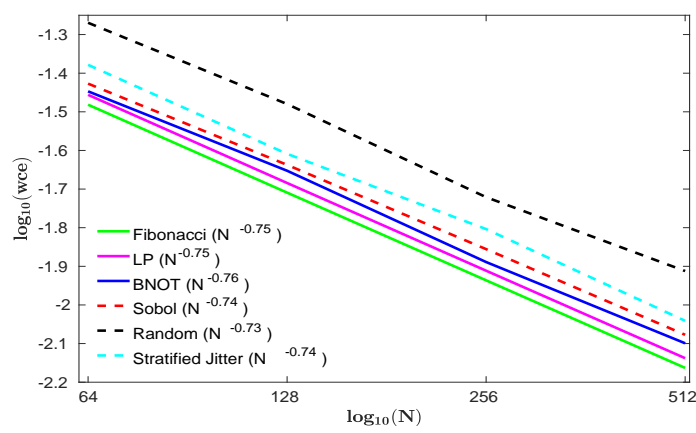

(b) With optimal sample weights

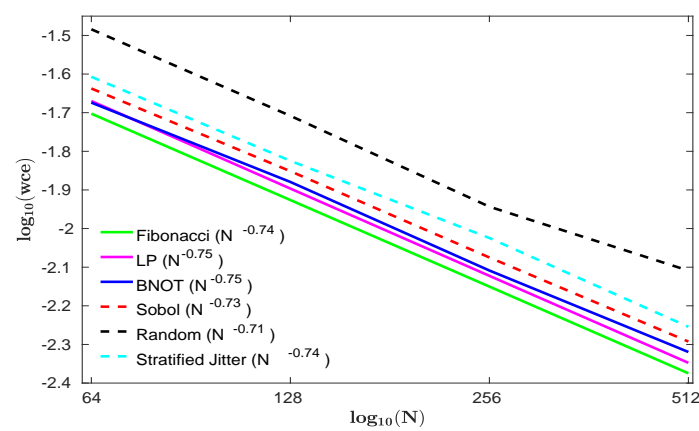

(d) With optimal sample weights

Fig. 4. Theoretical worst-case error, wce, (Eq. (42)) as a function of the number of samples $N$. The results are presented for equal weights (left column) and optimal weights (right column) quadrature rules using different point set generators. The top row shows the results for the diffuse reflection case, while the bottom row show the results for the glossy case using a shininess coefficient $n=30$. All results have been generated considering a Sobolev space smoothness coefficient $s=1.5$. For the sake of readability the results for the Random point set have been multiplied by a factor of 0.5 (respectively 0.45 ) in the top left plot (respectively bottom left), yielding $\log _{10}($ wce $)=\log _{10}($ Random $)-0.3\left(\right.$ respectively $\log _{10}($ wce $)=\log _{10}($ Random $\left.)-0.35\right)$. The original raw data can be found in the additional material. 


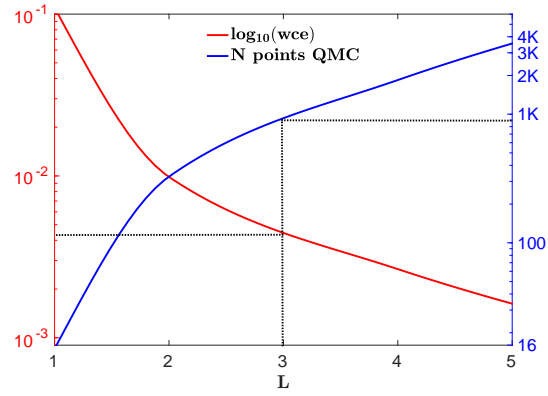

(a) Diffuse case with $\mathrm{SH}$

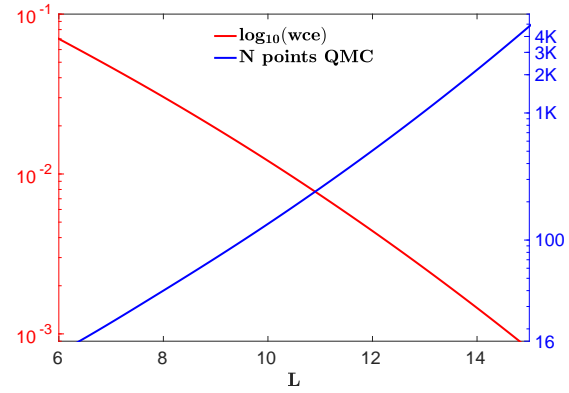

(b) Glossy case with SH

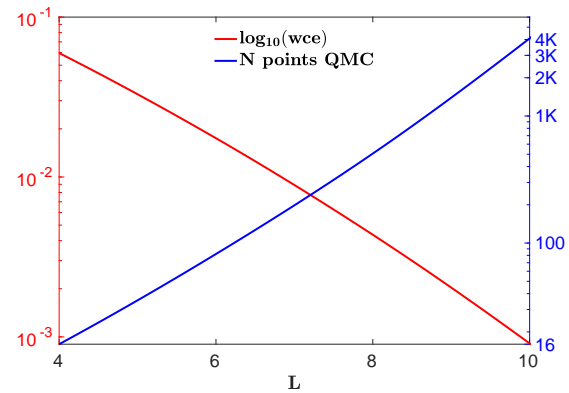

(c) Glossy case with HSH

Fig. 5. Worst case error analysis for the inner product truncation method in the diffuse and glossy reflection cases, considering that the incident radiance function $f(\mathbf{u})$ (see Eq. 17) belongs to a Sobolev space setting with $s=1.5$. The shininess coefficient for the glossy reflection case is $n=30$. In each plot, the scale for the red and blue lines is given by the left and right y-axis, respectively. The red lines depict the logarithm of the worst-case error wce as a function of the cutoff frequency $L$. For example, in (a), the worst-case error for $L=3$ is $\approx 4.4 \times 10^{-3}$. The blue lines represent the number of points required by QMC (using equal weights and a spherical Fibonacci point set) to achieve the same worst-case error as the inner product truncation method. For example, in (a), QMC requires $\approx 930$ samples to reach the same worst-case error as the truncated inner product method for $L=3$. (a) and (b) assume spherical harmonics $(\mathrm{SH})$ representation of $f(\mathbf{u})$ while in $(\mathbf{c}) f(\mathbf{u})$ is represented by hemispherical harmonics $(\mathrm{HSH})$.

have a rate of decay in $O\left(l^{-2 s}\right)$, the 'ideal' quadrature rule should thus have low values of $\left(d_{l}^{2}+C_{l}^{2}\right)$ coefficients at low frequencies, so as to yield the lowest possible approximation error. A more detailed analysis on this issue is provided in Sec. 7.2. Finally, noting that the $d_{l}$ coefficients are zonal (i.e., invariant to rotation about the $z$-axis and only dependent on the degree $l$ of the $\mathrm{SH}$ ) and that the $C_{l}$ coefficients are anisotropic, the worst-case error as defined by Eq. (42) can be split into zonal error $E_{Z}^{(s)}$ and anisotropic error $E_{A}^{(s)}$, such that $E^{(s)}=\left(E_{Z}^{(s)}+E_{A}^{(s)}\right)^{1 / 2}$, where:

$$
\left(E_{Z}^{(s)}\right)^{2}=\sum_{l=0}^{\infty} a_{l}^{(s)} d_{l}^{2}, \quad \text { (46) } \quad\left(E_{A}^{(s)}\right)^{2}=\sum_{l=1}^{\infty} a_{l}^{(s)} C_{l}^{2} .
$$

This formulation of the worst-case error is useful for the analysis made in Sec. 7.2 since, as we will show later, the error of QMC quadratures is essentially dominated by the anisotropic component $E_{A}^{(s)}$.

\subsection{Fourier domain inner product truncation}

Comparing Eq. (19) with Eq. (35), we can interpret Eq. (35) as the inner product on $\mathbb{L}^{2}$ of $f(\mathbf{u})$ with an approximate weight function $p^{\prime}(\mathbf{u})$ obtained by truncating the $\mathrm{SH}$ expansion of $p(\mathbf{u})$ :

$$
p^{\prime}(\mathbf{u})=2 \pi r_{s} \sum_{l=0}^{L} b_{l}^{(p)} Y_{l, 0}(\mathbf{u})
$$

The same equation applies to the HSH expansion case by replacing $b_{l}^{(p)}$ and $Y_{l, 0}$ with $\tilde{b}_{l}^{(p)}$ and $\tilde{Y}_{l, 0}$ respectively. However, $f(\mathbf{u})$ is still defined on the whole sphere $\mathbb{S}^{2}$ and so are the Sobolev spaces $\mathbb{H}^{s}\left(\mathbb{S}^{2}\right)$. Therefore, in the Fourier domain, the inner product of Eq. (39) is basically expanded in SH basis functions as defined by Eq. (12). So as to make use of Eq. (12) in the case of truncation in the HSH expansion, the set of HSH coefficients $\left\{\tilde{b}_{l}^{(p)}\right\}, l \in[0, L]$ has to be converted in SH coefficients $b_{l}^{(p)}$ as explained in section 4.4. Note that in this case, the converted SH expansion is then no longer limited to $L$ coefficients.

In Sec. 6.2 we show that, for the inner product truncation method, the representer of the approximation error as defined by Eq. (36) is given by:

$$
h^{(s)}(\mathbf{u})=\frac{1}{2 \pi r_{s}} \int_{S^{2}} K^{(s)}(\mathbf{u}, \mathbf{v})\left[p(\mathbf{v})-p^{\prime}(\mathbf{v})\right] d S(\mathbf{v})
$$

and that the worst-case error can be expressed as follows:

$$
E^{(s)}=\left(\int_{S^{2}} h^{(s)}(\mathbf{u})\left[p(\mathbf{u})-p^{\prime}(\mathbf{u})\right] d S(\mathbf{u})\right)^{1 / 2}
$$

which can be expanded in terms of SH coefficients with:

$$
E^{(s)}=\left(\sum_{l=0}^{\infty} a_{l}^{(s)}\left[b_{l}^{(p)}-b_{l}^{(p)}\right]^{2}\right)^{1 / 2}
$$

where the $b_{l}^{\prime(p)}$ are the SH coefficients of $p^{\prime}(\mathbf{u})$. When the truncation is performed on the $\mathrm{SH}$ expansion of the inner product (cf. Eq. (35)), $b_{l}^{(p)}=b_{l}^{(p)}$ for $l \in[0, L]$ and $b_{l}^{\prime(p)}=0$ otherwise and the worst-case error is then:

$$
E^{(s)}=\left(\sum_{l=L}^{\infty} a_{l}^{(s)}\left[b_{l}^{(p)}\right]^{2}\right)^{1 / 2}
$$

The above equation forms the core of our contribution to the spectral analysis of the error of inner product truncation methods. When the truncation is performed on the HSH expansion, the $b_{l}^{(p)}$ are obtained by converting the $L \mathrm{HSH}$ coefficients $\tilde{b}_{l}^{(p)}$ into $\mathrm{SH}$ coefficients as indicated above.

\section{Proofs of the spectral distribution of the error}

\subsection{Proof for the quadrature case}

The goal of this section is to prove Eq. (42), which expresses the worst case error $E^{(s)}$ as a product between two sets of coefficients, i.e., the $a_{l}^{(s)}$ coefficients which characterize the smoothness of the integrand $f(\mathbf{u})$; and $d_{l}^{2}+C_{l}^{2}$, the power spectrum coefficients which characterize the quadrature rule. The starting point is the worst-case error expression for hemispherical quadratures given by Eq. (41). Using the spherical harmonics (SH) expansion of the Sobolev kernel $K^{(s)}(\mathbf{u}, \mathbf{v})$ (Eq. (11)), the first term of the sum in brackets in Eq. (41) can be expanded in terms of spherical harmonics as follows:

$$
\sum_{i=1}^{N} \sum_{j=1}^{N} w_{i} w_{j} K^{(s)}\left(\mathbf{u}_{\mathbf{i}}, \mathbf{u}_{\mathbf{j}}\right)=\sum_{l=0}^{\infty} a_{l}^{(s)} \sum_{m=-l}^{m=l}\left|\sum_{j=1}^{N} w_{j} Y_{l, m}\left(\mathbf{u}_{j}\right)\right|^{2} .
$$


Let $\left\{c_{l, m}\right\}$ be the set of spectral coefficients defined by:

$$
c_{l, m}=\sum_{j=1}^{N} w_{j} Y_{l, m}\left(\mathbf{u}_{\mathbf{j}}\right) .
$$

We can then re-write Eq. (53) as:

$$
\sum_{i=1}^{N} \sum_{j=1}^{N} w_{i} w_{j} K^{(s)}\left(\mathbf{u}_{\mathbf{i}}, \mathbf{u}_{\mathbf{j}}\right)=\sum_{l=0}^{\infty} a_{l}^{(s)} \sum_{m=-l}^{m=l}\left|c_{l, m}\right|^{2} .
$$

Similarly, the second term of the sum in Eq. (41) can be expanded in terms of SH. After changing the order of the sums, this yields:

$$
\begin{aligned}
& \frac{1}{\pi r_{s}} \sum_{j=1}^{N} w_{j} \int_{S^{2}} K^{(s)}\left(\mathbf{u}_{\mathbf{j}}, \mathbf{u}\right) p(\mathbf{u}) d S(\mathbf{u}) \\
= & 2 \sum_{l=0}^{\infty} a_{l}^{(s)} \sum_{m=-l}^{m=l} \frac{1}{2 \pi r_{s}} \int_{S^{2}} Y_{l, m}^{*}(\mathbf{u}) p(\mathbf{u}) d S(\mathbf{u}) \sum_{j=1}^{N} w_{j} Y_{l, m}\left(\mathbf{u}_{j}\right) \\
= & 2 \sum_{l=0}^{\infty} a_{l}^{(s)} b_{l}^{(p)} c_{l, 0} .
\end{aligned}
$$

In the last line of the development, we use Eq. (20) and the fact that $p(\mathbf{u})$ is a zonal function. Consequently, only the zonal $\mathrm{SH}$ coefficients are non-zero. As for the last term of the sum in Eq. (41), Eq. (11) can be used again to expand the kernel in the same way as for the second term, which gives:

$$
\frac{1}{4 \pi^{2} r_{s}^{2}} \int_{S^{2}} \int_{S^{2}} K^{(s)}(\mathbf{u}, \mathbf{v}) p(\mathbf{u}) p(\mathbf{v}) d S(\mathbf{u}) d S(\mathbf{v})=\sum_{l=0}^{\infty} a_{l}^{(s)}\left(b_{l}^{(p)}\right)^{2} .
$$

Using the Eqs. (55), (56) and (57), we can re-write Eq. (41) as:

$$
\begin{aligned}
E^{(s)} & =\left[\sum_{l=0}^{\infty} a_{l}^{(s)}\left(\sum_{m=-l}^{m=l}\left|c_{l, m}\right|^{2}-2 b_{l}^{(p)} c_{l, 0}+\left(b_{l}^{(p)}\right)^{2}\right)\right]^{1 / 2} \\
& =\left[\sum_{l=1}^{\infty} 2 a_{l}^{(s)} \sum_{m=1}^{m=l}\left|c_{l, m}\right|^{2}+\sum_{l=0}^{\infty} a_{l}^{(s)}\left(c_{l, 0}^{2}-2 b_{l}^{(p)} c_{l, 0}+\left(b_{l}^{(p)}\right)^{2}\right)\right]^{1 / 2} \\
& =\left[2 \sum_{l=1}^{\infty} a_{l}^{(s)} \sum_{m=1}^{m=l}\left|c_{l, m}\right|^{2}+\sum_{l=0}^{\infty} a_{l}^{(s)}\left(b_{l}^{(p)}-c_{l, 0}\right)^{2}\right]^{1 / 2}
\end{aligned}
$$

Let us now define $\left\{C_{l}^{2}\right\}$ as the set of power spectrum coefficients such that:

$$
C_{l}^{2}=2 \sum_{m=1}^{m=l}\left|c_{l, m}\right|^{2}
$$

with $c_{l, m}$ being given by Eq. (54). Then, we can use Eqs. (59) and (43) to rewrite Eq. (58) as:

$$
E^{(s)}=\left(\sum_{l=1}^{\infty} a_{l}^{(s)} C_{l}^{2}+\sum_{l=0}^{\infty} a_{l}^{(s)} d_{l}^{2}\right)^{\frac{1}{2}}=\left(a_{0}^{(s)} d_{0}^{2}+\sum_{l=1}^{\infty}\left(C_{l}^{2}+d_{l}^{2}\right)\right)^{\frac{1}{2}},
$$

which proves Eq. (42). Finally, let us note that the $c_{l, 0}$ and $C_{l}^{2}$ coefficients can alternatively be expressed in terms of Legendre polynomials only. The $\mathrm{SH}$ of order $m=0$ are then reduced to:

$$
Y_{l, 0}(\theta, \phi)=\sqrt{\frac{(2 l+1)}{4 \pi}} P_{l}(\cos \theta),
$$

from which Eq. (45) is easily derived. As for the $C_{l}^{2}$ coefficients, the $\mathrm{SH}$ addition theorem (Eq. (3)) is used to derive Eq. (44) from Eqs. (54) and (59).

\subsection{Proofs for the inner product truncation case}

In the inner product truncation case, given the approximated weight function $p^{\prime}(\mathbf{u})$ resulting from the truncation of the Fourier expansion of $p(\mathbf{u})$, the approximated integral value is then:

$$
\tilde{I}_{f}=\frac{1}{2 \pi r_{s}} \int_{S^{2}} f(\mathbf{v}) p^{\prime}(\mathbf{v}) d S(\mathbf{v}) .
$$

The approximation error can then be expressed by:

$$
I_{f}-\tilde{I}_{f}=\frac{1}{2 \pi r_{s}} \int_{S^{2}} f(\mathbf{v})\left[p(\mathbf{v})-p^{\prime}(\mathbf{v})\right] d S(\mathbf{v}),
$$

Using Eq. (7), $f(\mathbf{v})$ can be replaced by its inner product formulation, which gives:

$$
I_{f}-\tilde{I}_{f}=\frac{1}{2 \pi r_{s}} \int_{S^{2}}\left\langle f(\cdot), K^{(s)}(\cdot, \mathbf{v})\right\rangle_{\mathbb{H}^{s}}\left[p(\mathbf{v})-p^{\prime}(\mathbf{v})\right] d S(\mathbf{v}) .
$$

As the inner product is a symmetric bilinear operator, we can rewrite the above equation as follows:

$$
I_{f}-\tilde{I}_{f}=\frac{1}{2 \pi r_{s}}\left\langle f(\cdot), \int_{S^{2}} K^{(s)}(\cdot, \mathbf{v})\left[p(\mathbf{v})-p^{\prime}(\mathbf{v})\right] d S(\mathbf{v})\right\rangle_{\mathbb{H}^{s}} .
$$

Noting that Eq. (64) has the same form as Eq. (36), we can easily identify $h^{(s)}(\mathbf{u})$ as stated in Eq. (49). Then, using Eq. (39), we can write the worst-case error $E^{(s)}$ for the inner product truncation case as:

$$
\begin{aligned}
E^{(s)}= & \left(\frac{1}{4 \pi^{2} r_{s}^{2}} \int_{S^{2}} \int_{S^{2}}\left\langle K^{(s)}(\mathbf{u}, .), K^{(s)}(\mathbf{v}, .)\right\rangle_{\mathbb{H}^{s}}\right. \\
& {\left.\left[p(\mathbf{u})-p^{\prime}(\mathbf{u})\right]\left[p(\mathbf{v})-p^{\prime}(\mathbf{v})\right] d S(\mathbf{u}) d S(\mathbf{v})\right)^{1 / 2} . }
\end{aligned}
$$

Using the symmetry property of the kernel, the inner product in the integral can be reduced to $K^{(s)}(\mathbf{u}, \mathbf{v})$, which gives:

$$
\begin{aligned}
E^{(s)}= & \left(\frac{1}{4 \pi^{2} r_{s}^{2}} \int_{S^{2}} \int_{S^{2}} K^{(s)}(\mathbf{u}, \mathbf{v})\right. \\
& {\left.\left[p(\mathbf{u})-p^{\prime}(\mathbf{u})\right]\left[p(\mathbf{v})-p^{\prime}(\mathbf{v})\right] d S(\mathbf{u}) d S(\mathbf{v})\right)^{1 / 2} . }
\end{aligned}
$$

At this point, we can reuse the result given by Eq. (57) above. Indeed, by substituting $p(\mathbf{u})-p^{\prime}(\mathbf{u})$ for $p(\mathbf{u})$ and $p(\mathbf{v})-p^{\prime}(\mathbf{v})$ for $p(\mathbf{v})$ in Eq. (57) above, we obtain the same expression as in Eq. (66). We can thus use Eq. (57) in which we substitute $\left(b_{l}^{(p)}-b_{l}^{(p)}\right)$ for $b_{l}^{(p)}$ to get Eq. (51).

\section{Analysis of COMmonLy-used QMC methods}

\subsection{Our QMC test set}

In this subsection, we apply the theoretical framework presented above to analyze the spectral characteristics of the quadrature error produced by the QMC method. Two sample weighting methods are tested: (i) the typical QMC case where the $w_{j}$ weights are equal to $1 / N$, with $N$ being the number of samples; and the optimal sample weights (OSW) of Marques et al. [19], where the sample weights are determined by explicitly minimizing the worst case integration error of Eq. (41). Six sample sets are analyzed:

- $\quad$ Sobol $(0,2)$-sequence [28];

- Periodic blue noise though optimal transport (BNOT) [8];

- Larcher-Pillichshammer (LP) point set [18];

- Spherical Fibonacci (SF) [12], [20];

- A Random point set;

- A Stratified Jitter (SJ) point set. 
The SF point set is directly defined on the hemisphere while the other point sets are generated on the unit square and lifted to the unit hemisphere using a Lambert cylindrical equal-area projection. In the following subsections, the spectral characteristics of the selected QMC methods are analyzed for the diffuse and glossy weight function presented in section 4 . Our goal in this analysis is not to carry out an exhaustive comparison of the performances of the selected QMC methods but to show how to use our framework to analyze the spectral distribution of error and to improve their efficiency. For this purpose, we have chosen six point set generators: three of them are deterministic (Sobol, LP and $\mathrm{SF}$ ) while the other three (BNOT, Random and SJ) are stochastic.

\subsection{Spectral analysis of the selected QMC methods}

In Sec. 5.1, we have expressed the spectral distribution of the worst-case error in a Sobolev setting under the form of Eq. (42). In the following, this equation is used to analyze the spectral distribution of error for various combinations of QMC sequences and integrand weight functions. For the sake of argument, it is interesting to first examine the power spectrum of the quadrature rule alone, as expressed by the zonal and anisotropic power spectrum coefficients coefficients $d_{l}^{2}$ and $C_{l}^{2}$. These power spectra are shown in Fig. 1. Let us first focus on the equal-weights quadrature for the diffuse case (Figs. 1(a) and 1(b)). When comparing both spectra it clearly appears that, in general, the zonal power spectrum coefficients $d_{l}^{2}$ are much smaller than the anisotropic power spectrum coefficients $C_{l}^{2}$, which means that the contribution of the zonal error $E_{Z}^{(s)}$ (Eq. (46)) to the total error is very small. In Fig. 1(c), one can appreciate the effect of the optimal sample weights (OSW [19]) which act by reducing the coefficients at the lower frequencies (see also Fig. 2 for a more detailed comparison). As explained in Sec. 5.2, it is in the lower frequencies that the $a_{l}^{(s)}$ coefficients characterizing the incident radiance function have a larger value. Therefore, to minimize the worst-case error, it is optimal to reduce the quadrature power spectrum coefficients at the lower frequencies, so as to minimize the product in Eq. (42). Figs. 1(d) to 1(f) show that the power spectrum coefficients in the glossy case exhibit the same characteristics.

The power spectrum of the quadrature rule alone is not sufficient to characterize the spectral distribution of the quadrature error. Information on the integrand spectrum is necessary for a more complete analysis of the quadrature error. As we have seen in section 5.1, this information can be accounted for by choosing an appropriate Sobolev space setting, hence the weights sequence $a_{l}^{(s)}$ used to weight the quadrature rule power spectrum coefficients in Eq. (42). In the following, we analyze the spectral distribution of error as given by the power spectrum coefficients $e_{l}^{2}=a_{l}^{(s)}\left(d_{l}^{2}+C_{l}^{2}\right)$. In all cases the used smoothness parameter is $s=1.5$ which proves to be an appropriate function space to model incident radiance functions (c.f. Sec. 9). We have chosen to distribute our samples using importance sampling according to $\operatorname{BRDFs}(p(\mathbf{u}))$ as it is the common practice in rendering. This approach, which henceforth is referred to as morphing, makes the sampling density mimic better the integrand and can be applied to deterministic as well as stochastic samples sets.

In the diffuse reflection case, all tested point sets are morphed with the classic $z=\sqrt{z^{\prime}}$ function to implement importance sampling. The resulting spectral distributions are shown in Figs 3(a) and 3(b) for equal-weights and optimal-weights quadratures, respectively. As expected, the contribution of high frequency components is strongly reduced by the decay in $O\left(l^{-2 s}\right)$ of the $a_{l}^{(s)}$ coefficients. On the contrary, some low frequency components that are undetectable in Figs. 1(b) and 1(c) appear to have a much higher contribution when multiplied by the $a_{l}^{(s)}$ coefficients. Note also the strong effect of the OSW technique [19], which drastically reduces the power spectrum of the error at low frequencies.

The spectral distribution of error in the glossy reflection case is shown in Figs. 3(c) and 3(d). To implement importance sampling, the morphing function for the Gaussian weight function is [21]:

$$
z=\frac{1}{n} \ln \left[1+z^{\prime}\left(e^{n}-1\right)\right]
$$

where $n$ is the glossiness (Eq. (25)). The spectral extent of the anisotropic error component covers a much wider frequency range than for the diffuse case. This illustrates quite well why the shading integral for the glossy reflection case is much more sensitive to high-frequencies content than for the diffuse case. The effect of the OSW is also well noticeable in the glossy case, as the low frequency components of the error are reduced whereas the high frequency components are left unchanged. This explains why the OSW are more effective on smooth integrands for which the spectrum tends to concentrate on low frequency components.

Finally, Fig. 4 shows the theoretical worst-case error as a function of the number of samples. It shows that the point sets which exhibit higher power spectra coefficients in Fig. 3 consistently yield a larger worst-case error value. It also confirms that, in theory, a good sampling pattern must have the lowest possible power spectrum in the low frequencies. Indeed, the point sets exhibiting these features in Figs. 1 and 2 are those with the best performance in terms of worst-case error values in Fig. 4 . The effect of error reduction provided by the use of the OSW is also clearly visible in Fig. 4, especially for the Random (and thus less evenly distributed) point sets. In particular, let us point out that the use of the OSW improves the convergence rate of the Random point set, from the typical $N^{-0.5}$ to $N^{-0.75}, N$ being the number of samples. This theoretical result is in-line with previous evidence from Marques et al. [19] and will be further confirmed by our experimental results.

\section{Error analysis of INNER PRODUCt truncation MEthod}

In this section, we analyze the integration error, cf. Sec. 5.3, resulting from the inner product truncation. We consider a diffuse and a glossy reflectance function $p(\mathbf{u})$, and the Sobolev smoothness parameter is set to $s=1.5$. Moreover, we compare this inner product truncation method to the classic QMC quadrature by considering the number of QMC sample points required to achieve the same worst-case error for both methods. For this comparison, we have used spherical Fibonacci point sets for the QMC method. For the inner product truncation method, we consider $\mathrm{SH}$ as well as HSH expansion cases. In the case of HSH, we suppose that the $\mathrm{HSH}$ coefficients of the integrand $f(\mathbf{u})$ are known precisely and the HSH rotation process is assumed error-free [11].

\subsection{Diffuse reflection case}

Fig. 5(a) shows the worst-case error results for the diffuse reflection case when the truncation is performed on the $\mathrm{SH}$ expansion. The red line shows that the worst-case error curve exhibits a bend at about $L=2$ beyond which the rate of decrease of the error is much slower. We can thus conclude that a sensible choice for the cutoff frequency is $L=3$. Still in Fig. 5(a), the blue line shows the number of QMC points required to reach the same worst-case error as with the inner product truncation method, for a given 
TABLE 1

Cutoff frequency $L$ and equivalent number of QMC points $N$ required to reach a worst-case error $E^{(1.5)}=2.5 \times 10^{-3}$, with an integrand smoothness $s=1.5$.

\begin{tabular}{c|c|c|c} 
& Diffuse SH & Glossy SH & Glossy HSH \\
\hline Cutoff Frequency & $L=4$ & $L=13$ & $L=9$ \\
\hline Equivalent QMC points & 2000 & 1000 & 1000
\end{tabular}

cutoff frequency $L$. It can be seen that QMC quickly requires a large number of samples to achieve a similar error value, even when $L$ is kept at relative small values such as 3 or 4 . This is because, for this BRDF case, the low frequency SH coefficients carry most of the information required to perform a good quality integral estimate. However, as we show below, the SH Fourier truncation method becomes less efficient in the glossy case. In the HSH expansion case, exact integration is obtained with $L=2$ since, as shown in Sec. 4.2, the HSH coefficients of the diffuse reflectance function have a value $\tilde{b}_{l}^{(d)}=0$ for $l>2$ (see also Eq (51)).

\subsection{Glossy reflection case}

In the glossy reflection case, the error decrease is almost linear in log scale as shown in Fig. 5(b). For an equal worst-case error value, the cutoff frequency must be significantly higher in the glossy reflection case than for the diffuse case. For example, using $L=4$ is sufficient to reach a worst-case error of 0.0025 in the diffuse reflection case whereas $L=13$ is necessary to reach the same worst-case error in the glossy reflection case with a glossiness $n=30$. Moreover, comparing Fig. 5(a) with Fig. 5(b), we can see that the same number of QMC samples is equivalent to a much larger number of SH coefficients in the glossy case. Our theoretical results also show the benefits of the use of HSH instead of $\mathrm{SH}$, as shown in Fig. 5(c). Note the higher rate of decrease of the error with HSH compared to SH. For the same worst-case error value $E^{(1.5)}=10^{-2}$, the cutoff frequency reaches $L \approx 11$ for $\mathrm{SH}$ and $L \approx 7$ for $\mathrm{HSH}$, hence saving an important number of coefficients. The above results are summarized in Table 1.

\section{Experimental results}

In this section we present a set of experimental results related to real evaluations of the rendering integral. The section is divided in two subsections: the first one aims at validating the choice of the used Sobolev space parameter $s=1.5$ and its appropriateness to model incident radiance functions; the second shows that the experimental error found in our experiments is in accordance with the theoretical results presented in Secs. 7 and 8.

\subsection{Sobolev space coefficient}

Fig. 6 shows the result of reconstructing the original environment map using three different values of shininess parameter $s$ and 20000 samples through kernel-based interpolation (see e.g. Fasshauer and McCourt [9]). The kernel used for the interpolation is given by Eq. (14). By examining each of the Figs. 6(a) to $6(\mathrm{c})$, and comparing with the original environment map in Fig. 6(d), one can observe that the reconstruction which better preserves the image details is the one with $s=1.5$. This subjective superior quality is confirmed by the last column of Tab. 2, which shows that the root mean squared error (RMSE) of the regression performed using $s=1.5$ is $18 \%$ lower than that of $s=1.1$ and $20 \%$ than that of $s=1.1$. Similar observations can be made for

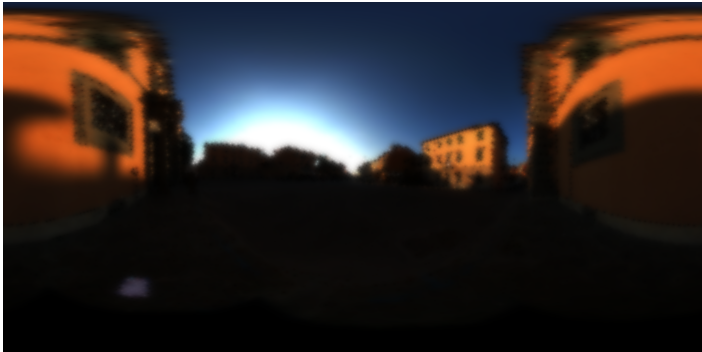

(a) $s=1.1$

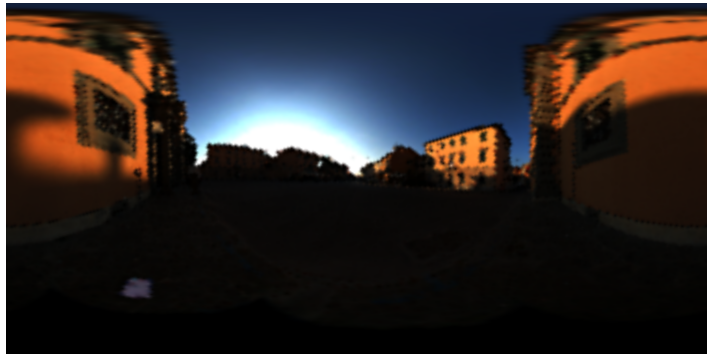

(b) $s=1.5$

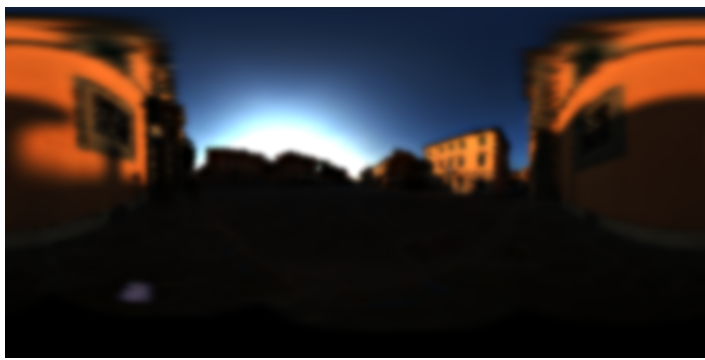

(c) $s=1.9$

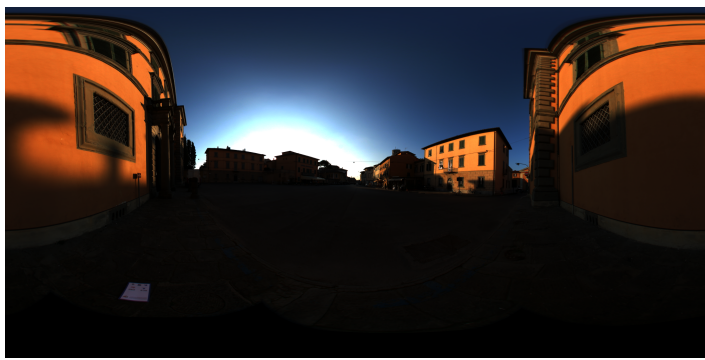

(d) Original

Fig. 6. Reconstruction an the original environment map (d) using a kernel regression with three different values for the Sobolev coefficient $s .20000$ samples were used in each of the cases. Note that using $s=1.5$ yields the results which are visually closer to the original environment map. These visual clues supporting the appropriateness of the choice of $s=1.5$ are confirmed by the quantitative results in Tab. 2. Results for another environment map can be found in additional material.

lower sample counts. Results for another environment map can be seen in additional material.

We have observed that, in most cases of lighting environments, the smoothness parameter that yields the minimal approximation RMSE is quite close to $s=1.5$, hence our choice to focus on the $s=1.5$ case in the error analysis considered in Secs. 7 and 8 .

\subsection{Experimental error analysis}

In the following, we give numerical evidence for the theory developed in this paper. To this end, we have evaluated a large quantity of rendering integrals using the QMC method of Eq. (34) and 


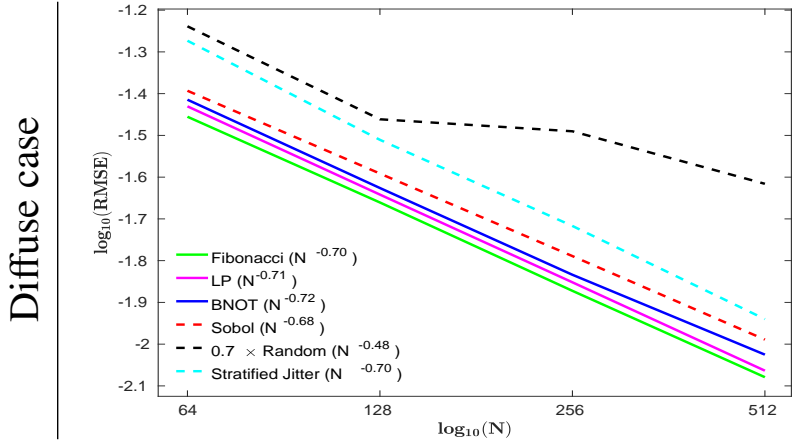

(a) Standard QMC (equal weights)

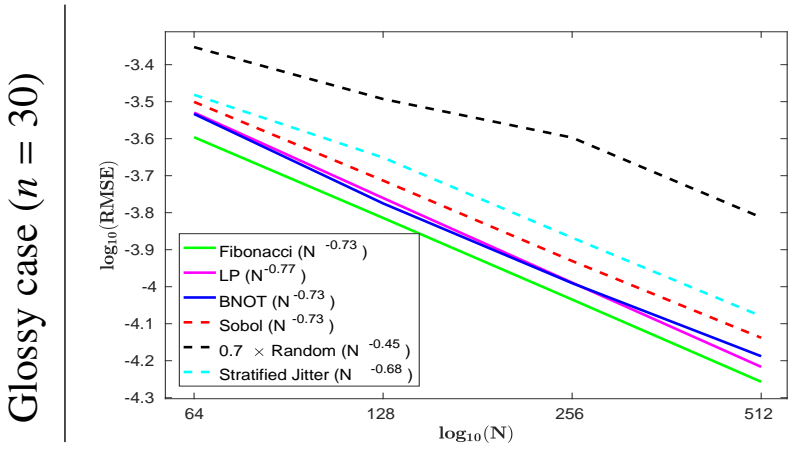

(c) Standard QMC (equal weights)

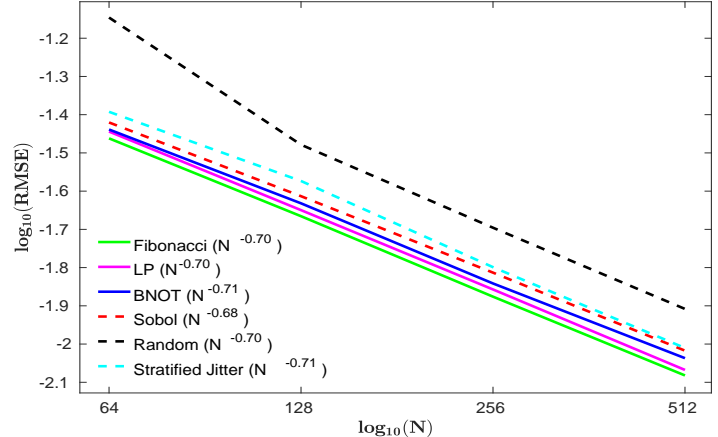

(b) With optimal sample weights (OSW)

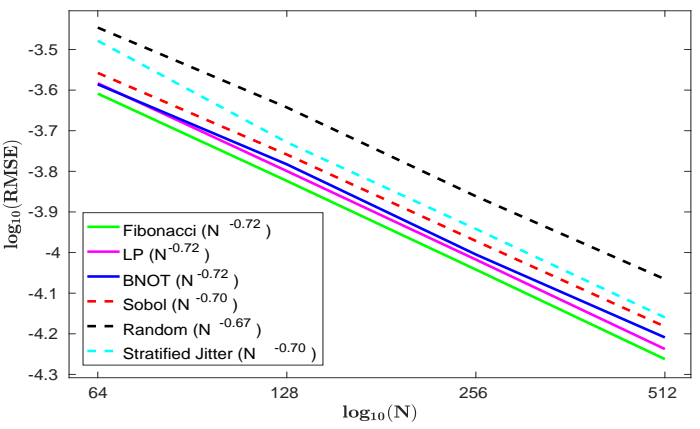

(d) With optimal sample weights (OSW)

Fig. 7. Experimental root mean squared error (RMSE) values for QMC method as a function of the number of samples $N$, for the different tested point sets. The incident radiance function is given by the Pisa environment map. For each point set type of size $N$, the RMSE has been computed by considering a very large number of randomly generated spherical directions. Each of these directions is used as the vertical axis of a hemisphere over which the rendering integral is evaluated. To this end, we sample the incident radiance function by tracing $N$ rays, the directions of which are given by the current point set. We then use these samples to compute the QMC estimate (c.f. Eq. (34)), and compare it to the reference solution. The top and bottom rows depict the diffuse and glossy reflection cases, respectively. The shininess coefficient for the glossy reflection case is $n=30$. The left column shows the results obtained using an equal-weights quadrature, while the right column shows the results for the optimal sample weights (OSW) case. For the sake of readability the results for the Random point set have been multiplied by a factor of 0.7 in the left column, yielding $\log _{10}(\mathrm{RMSE})=\log _{10}(\mathrm{Random})-0.15$. The original raw results can be found in additional material.

TABLE 2

RMSE of the interpolated PISA environment maps.

\begin{tabular}{c||ccccccc}
\multicolumn{1}{c||}{} & \multicolumn{7}{c}{ \# Samples } \\
\cline { 2 - 8 }$s$ & 100 & 500 & 1000 & 2500 & 5000 & 10000 & 20000 \\
\hline 1.1 & 0.255 & 0.174 & 0.156 & 0.130 & 0.114 & 0.098 & 0.085 \\
1.5 & 0.234 & 0.152 & 0.138 & 0.114 & 0.098 & 0.083 & 0.070 \\
1.9 & 0.241 & 0.153 & 0.139 & 0.120 & 0.107 & 0.097 & 0.089
\end{tabular}

the Fourier truncation method of Eq. (35). The incident radiance function $f(\mathbf{u})$ in Eq. (17) is given by an environment map. For the QMC method, both the equal-weights and the OSW variants have been tested. Fig. 7 shows the error plots for the quadrature rule method using the Pisa environment map of Fig. 6(d). The results are in accordance with the theoretical worst-case error plots of Fig. 4 up to a multiplicative factor which has to do with the norm of $f(\mathbf{u})$ (recall that this was assumed to be $\|f(\mathbf{u})\|=1$ in our theoretical developments). This shows that our theoretical framework can be used to evaluate the relative performance of different quadrature rule configurations (i.e., the quality of the samples distribution and of the associated sample weights). Moreover, Fig. 7 also shows that our framework succeeded in predicting that the convergence rate of the random point set when using OSW is $\approx N^{-0.7}$ as opposed to $\approx N^{-0.5}$ with equal weights. These results are inline with evidence from Marques et al. [19], and are also an indication that a Sobolev space with a smoothness parameter $s=1.5$ is, in general, an appropriate function space to represent incident radiance functions. The experimental results for the Fourier domain inner product truncation method, shown in Fig. 8, also confirm the theoretical error prediction made by our framework. Indeed, for all three cases analyzed, the experimental error results are roughly similar to those predicted in Fig. 5. It is interesting to note that our framework rather well predicts the equivalence (as defined in Sec. 8), in terms of approximation error, between the QMC method and the inner product truncation method.

\section{Conclusion}

We have shown that the theoretical framework proposed in this paper allows analyzing the spectral characteristics and predicting the performances of quadrature rules applied to shading integrals. Our theoretical approach is based on the theory of Sobolev spaces, which enables us to model the smoothness of integrands. This approach allows analyzing the spectral characteristics of quadrature rules in a more comprehensive manner than simply considering the sampling patterns spectrum as it is usually done in the current QMC literature. Furthermore, we have taken into account the specificities of shading integrals by considering hemispheric integration domains and integrands that include a BRDF model under the form of an analytically-known weight function. We have clearly shown that our approach is advantageous compared 


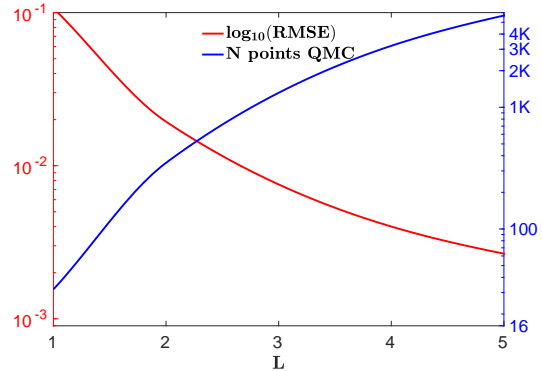

(a) Diffuse case with $\mathrm{SH}$

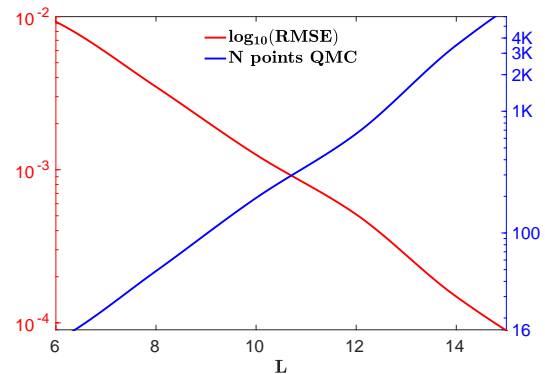

(b) Glossy case with SH

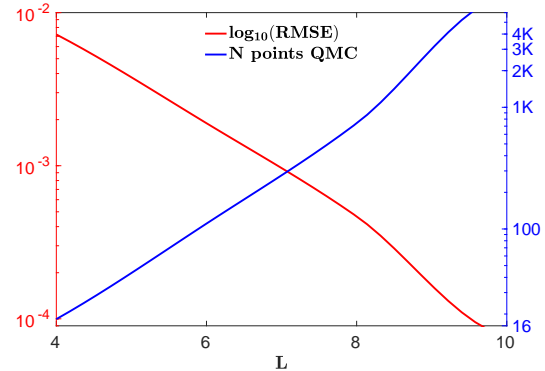

(c) Glossy case with HSH.

Fig. 8. Experimental root mean squared error (RMSE) for the inner product truncation method in the diffuse and glossy reflection cases. The incident radiance function is given by the Pisa environment map. For each point set type of size $N$, the RMSE has been computed by considering a very large number of randomly generated spherical directions. Each of these directions is used as the vertical axis of a hemisphere over which the rendering integral is evaluated. So as to compute the shading integral for each of these directions, we determine the incident radiance function SH/HSH coefficients $f_{l m}$ taking as vertical axis the current direction. We then compute the integral estimate using Eq. (35), and compare it to the reference solution. The shininess coefficient for the glossy reflection case is $n=30$. The scale for the red and blue lines are given by the left and right $y$-axis, respectively. The red lines depict the logarithm of the RMSE as a function of the cutoff frequency $L$. The blue lines represent the number of points required by QMC (using equal weights and a spherical Fibonacci point set) to achieve the same RMSE as the inner product truncation method. (a) and (b) assume spherical harmonics $(\mathrm{SH})$ representation of $f(\mathbf{u})$ while in (c) $f(\mathbf{u})$ is represented by hemispherical harmonics $(\mathrm{HSH})$.

to previous work. More specifically: (1) our approach takes into account the BRDF and importance sampling (morphing), and we show that these two factors have a strong effect on the spectral analysis of the worst-case error; (2) taking into account the samples weights enables us to clearly explain the effect on the spectral distribution analysis of the optimal sample weight technique; (3) we have also extended our analysis to the important case of inner product truncation technique in the spectral domain, which is crucial for global illumination methods such as Radiance Caching and Precomputed Radiance Transfer; (4) our theoretical framework is rather general regarding the smoothness assumption of the incident radiance, which allows the analysis a wide range of integrand smoothness. Lastly, the comparison of our theoreticallybased results with experimental rendering results shows that our theoretical model is valid and applicable to 3D scene rendering.

\section{Acknowledgements}

Ricardo Marques was supported by the European Union's Horizon 2020 research programme through a Marie Sklodowska-Curie Individual Fellowship (grant number 707027).

\section{References}

[1] M. Abramowitz and I. Stegun. Handbook of Mathematical Functions. Dover Publications, 1965.

[2] K. Atkinson and W. Han. Theoretical Numerical Analysis : a Functional Analysis Framework. Texts in applied mathematics. Springer, Dordrecht, New York, 2009.

[3] R. Basri and W. Jacobs. Lambertian Reflectance and Linear Subspaces. IEEE Trans. Pattern Anal. Mach. Intell., 25(2):218-233, February 2003.

[4] L. Belcour, C. Soler, K. Subr, N. Holzschuch, and F. Durand. 5D Covariance Tracing for Efficient Defocus and Motion Blur. ACM Trans. Graph., 32(3):31:1-31:18, July 2013.

[5] J. Brauchart and J. Dick. QMC Rules for Numerical Integration Over the Unit Sphere $\mathbb{S}^{2}$. Numer. Math., 121(3):473-502, July 2012

[6] J. Brauchart and P. Grabner. Distributing Many Points on Spheres. J. Complex., 31(3):293-326, June 2015.

[7] J. Brauchart, E. Saff, I. Sloan, and R. Womersley. QMC Designs: Optimal Order Quasi Monte Carlo Integration Schemes on the Sphere. Math. Comput., 83(290), 2014.

[8] F. de Goes, K. Breeden, V. Ostromoukhov, and M. Desbrun. Blue Noise through Optimal Transport. ACM Trans. Graph. (SIGGRAPH Asia), 31, 2012.
[9] G. Fasshauer and M. McCourt. Kernel-based Approximation Methods using MATLAB. World Scientific Publishing, 2016.

[10] W. Freeden and T. Gervens. Constructive Approximation on the Sphere: With Applications to Geomathematics. Numerical Mathematics and Scientific Computation. Clarendon Press, 1998.

[11] P. Gautron, J. Krivanek, S. Pattanaik, and K. Bouatouch. A Novel Hemispherical Basis for Accurate and Efficient Rendering. In Proceedings of the Fifteenth Eurographics Conference on Rendering Techniques, EGSR'04, pages 321-330, Aire-la-Ville, Switzerland, Switzerland, 2004. Eurographics Association.

[12] J. Hannay and J. Nye. Fibonacci Numerical Integration on a Sphere. Journal of Physics A: Mathematical and General, 37(48):11591, 2004.

[13] K. Hesse and I. Sloan. Cubature Over the Sphere in Sobolev Spaces of Arbitrary Order. Journal of Approximation Theory, 141(2):118 - 133, 2006.

[14] F. Hickernell. Quadrature Error Bounds with Applications to Lattice Rules. SIAM J. Numer. Anal., 33(5):1995-2016, October 1996.

[15] A. Keller. Quasi-Monte Carlo Image Synthesis in a Nutshell. In Monte Carlo and Quasi-Monte Carlo Methods 2012, volume 65 of Springer Proceedings in Mathematics $\mathcal{E}$ Statistics, pages 213-249. Springer Berlin Heidelberg, 2013

[16] R. Kennedy, P. Sadeghi, Z. Khalid, and J. McEwen. Classification and Construction of Closed-form Kernels for Signal Representation on the 2Sphere. In Proc. SPIE 8858, Wavelets and Sparsity XV, pages 88580M$88580 \mathrm{M}-15$. SPIE, 2013.

[17] J. Křivánek, P. Gautron, S. Pattanaik, and K. Bouatouch. Radiance Caching for Efficient Global Illumination Computation. Visualization and Computer Graphics, IEEE Transactions on, 11(5):550 -561, sept.oct. 2005.

[18] G. Larcher and F. Pillichshammer. Walsh Series Analysis of the L2Discrepancy of Symmetrisized Point Sets. Monatshefte für Mathematik, 132(1):1-18, 2001.

[19] R. Marques, C. Bouville, and K. Bouatouch. Optimal Sample Weights for Hemispherical Integral Quadratures. Computer Graphics Forum, 38(1):59-72, 2018.

[20] R. Marques, C. Bouville, M. Ribardière, L. P. Santos, and K. Bouatouch Spherical Fibonacci Point Sets for Illumination Integrals. Computer Graphics Forum, 32(8):134-143, December 2013.

[21] R. Marques, C. Bouville, M. Ribardière, L. P. Santos, and K. Bouatouch A Spherical Gaussian Framework for Bayesian Monte Carlo Rendering of Glossy Surfaces. IEEE Transactions on Visualization and Computer Graphics, 19(10):1619-1632, Oct 2013.

[22] S. Mehta, B. Wang, R. Ramamoorthi, and F. Durand. Axis-aligned Filtering for Interactive Physically-based Diffuse Indirect Lighting. ACM Trans. Graph., 32(4):96:1-96:12, July 2013.

[23] H. Nguyen and M. Do. Inverse Rendering of Lambertian Surfaces Using Subspace Methods. IEEE Trans. Image Processing, 23(12):5545-5558, 2014.

[24] A. Pilleboue, G. Singh, D. Coeurjolly, M. Kazhdan, and V. Ostromoukhov. Variance Analysis for Monte Carlo Integration. ACM Trans. Graph., 34(4):124:1-124:14, July 2015. 
[25] R. Ramamoorthi, J. Anderson, M. Meyer, and D. Nowrouzezahrai. A Theory of Monte Carlo Visibility Sampling. ACM Trans. Graph., 31(5):121:1-121:16, September 2012.

[26] Ravi Ramamoorthi and Pat Hanrahan. A Signal-processing Framework for Inverse Rendering. In Proceedings of the 28th Annual Conference on Computer Graphics and Interactive Techniques, SIGGRAPH '01, pages 117-128, New York, NY, USA, 2001. ACM, ACM.

[27] P. Sloan, J. Kautz, and J. Snyder. Precomputed Radiance Transfer for Real-Time Rendering in Dynamic, Low-Frequency Lighting Environments. ACM Transaction on Graphics, 21(3), 202.

[28] I. Sobol. On the Distribution of Points in a Cube and the Approximate Evaluation of Integrals. USSR Computational Math. and Math. Phys., 7:86-112, 1967.

[29] K. Subr and J. Kautz. Fourier Analysis of Stochastic Sampling Strategies for Assessing Bias and Variance in Integration. ACM Trans. Graph., 32(4):128:1-128:12, July 2013.

[30] J. Traub, G. Wasilkowski, and H. Woźniakowski. Information-based Complexity. Academic Press Professional, Inc., San Diego, CA, USA, 1988.

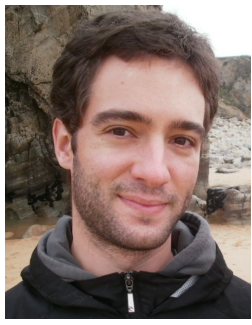

Ricardo Marques Ricardo Marques is Lecturer at the Universitat Pompeu Fabra (UPF), Barcelona. $\mathrm{He}$ received his MSc degree from University of Minho, Portugal (2009), after which he worked as a researcher in the same university. He joined INRIA (Institut National de Recherche en Informatique et Automatique) and the FRVSense team as a PhD student in the fall 2010 under the supervision of Kadi Bouatouch. In his thesis, he focused on spherical integration methods applied to light transport simulation. He defended his PhD thesis in the fall 2013 and joined the Mimetic INRIA research team as a research engineer in 2014, where he worked in the field of Crowd Simulation. From summer 2016 to summer 2018 he worked in the Interactive Technologies Group (GTI) of UPF as a post-doc, supported by a Marie Sklodowska-Curie individual fellowship.

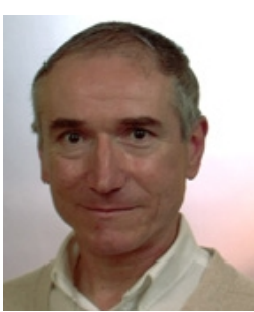

Christian Bouville Christian Bouville is presently invited researcher in the Percept team at IRISA (Institut de Recherche en Informatique et Systémes Aléatoires) in Rennes (France). He has been team leader, project leader and Emeritus expert at Orange Labs until 2006 and has been involved in many European and national projects. His main fields of research are now global illumination models and image perception with a special interest in machine learning approaches.

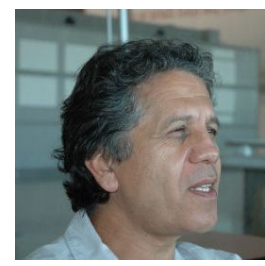

Kadi Bouatouch was an electronics and automatic systems engineer (ENSEM 1974). He was awarded a PhD in 1977 and a higher doctorate in computer science in the field of computer graphics in 1989. He is working on global illumination, lighting simulation for complex environments, GPU based rendering and computer vision. He is currently Professor at the University of Rennes 1 (France) and researcher at IRISA (Institut de Recherche en Informatique et Systémes Aléatoires). He is member of Eurographics. 